\title{
Error Analysis of a Finite Element-Integral Equation Scheme for Approximating the Time-Harmonic Maxwell System
}

\author{
G. C. Hsiao*, P. B. Monk ${ }^{\dagger}$, N. Nigam ${ }^{\ddagger}$
}

Technical Report No. 2002-06

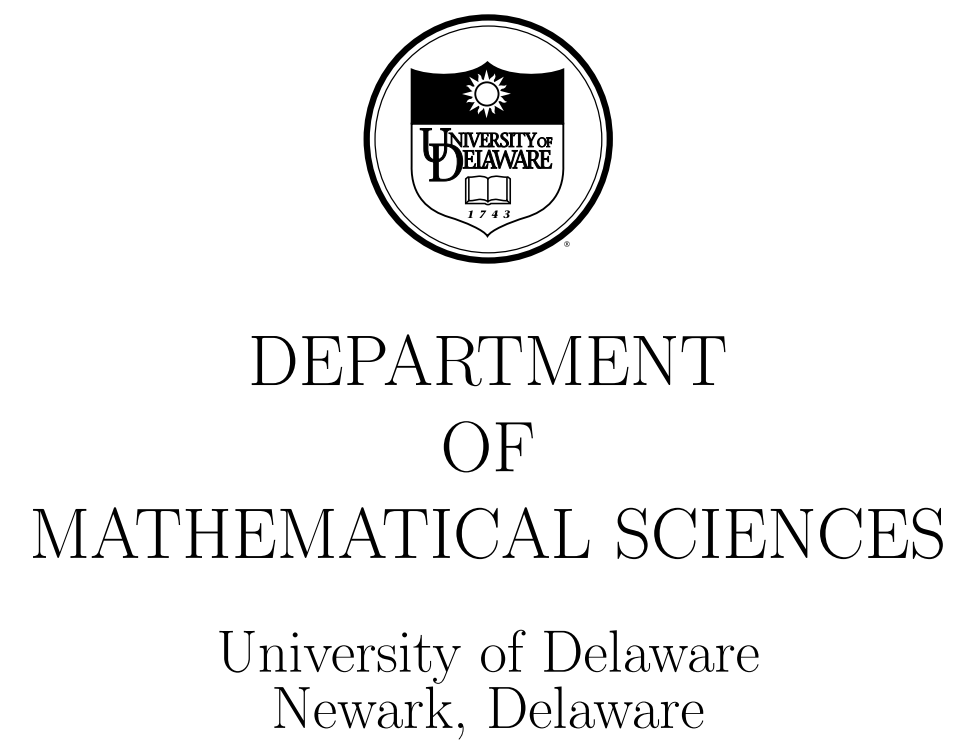

*Department of Mathematical Sciences, University of Delaware, Newark, DE 19716-2553 (hsiao@math.udel.edu).

$\dagger$ Department of Mathematical Sciences, University of Delaware, Newark, DE 19716-2553 (monk@math.udel.edu).

${ }^{\ddagger}$ IMA, University of Minnesota, Minneapolis, MN 55455 (nigam@ima.umn.edu). 


\title{
ERROR ANALYSIS OF A FINITE ELEMENT-INTEGRAL EQUATION SCHEME FOR APPROXIMATING THE TIME-HARMONIC MAXWELL SYSTEM
}

\author{
G. C. HSIAO* ${ }^{*}$ P. B. MONK ${ }^{\dagger}$, AND N. NIGAM ${ }^{\ddagger}$
}

\begin{abstract}
In 1996 Hazard and Lenoir suggested a variational formulation of Maxwell's equations using an overlapping integral equation and volume representation of the solution. They suggested a numerical scheme based on this approach, but no error analysis was provided. In this paper, we provide a convergence analysis of an edge finite element scheme for the method. The analysis uses the theory of collectively compact operators. It's novelty is that a perturbation argument is needed to obtain error estimates for the solution of the discrete problem that is best suited for implementation.
\end{abstract}

1. Introduction. A key feature of scattering problems is that they are typically posed as exterior boundary value problems. When using finite element methods to compute approximate solutions to these problems, the truncation of the computational domain needs to be done carefully. The truncated problem should be chosen to provide a convenient and accurate approximation of the true problem. In [25], Hazard and Lenoir proposed a new variational approach to the time-harmonic scattering problem for Maxwell's equations that can be used as the basis of a finite element method. This was extended to layered media in [19]. Hazard and Lenoir suggested the use of standard continuous finite elements, which are known to require special care if the scatterer has corners $([4,17,21])$. In this paper, we propose the use of edge elements $([31],[32])$ to approximate the problem. A direct application of this approach leads to unwieldy matrices. Thus we apply flux-recovery procedures $([35,5,6,7])$ in the discretization of the Hazard-Lenoir method resulting in a fully discrete problem that is better suited to implementation. We provide the first error analysis of the discrete Hazard-Lenoir scheme for Maxwell's equations (see [18] for convergence studies of the method applied to the time-harmonic Helmholtz problem).

For simplicity we will not describe the general scattering problem discussed in [19]. Instead, we shall confine ourselves to time-harmonic scattering from a bounded perfect conductor. The finite element scheme applies in the more general case, but some estimates in the analysis still need to be performed. The plan for the paper is as follows. In the next subsection we describe the continuous problem, and introduce a truncation based on an integral representation of the scattered field. In Section 2, we describe the finite elements to be used, and in Section 3 we provide an error analysis of the method without discretizing the integral operator. In Section 4 we provide an analysis of the fully discrete scheme and discuss briefly the solution of the discrete problem, and draw some conclusions in Section 5.

1.1. Problem description. We consider a perfectly conducting scatterer, which occupies a bounded, Lipschitz, polyhedral region $D$ in $\mathbb{R}^{3}$. We assume that the boundary of the scatterer, $\Gamma$, is connected and we denote by $\boldsymbol{\nu}$ the unit outward normal (see Figure 1.1). For simplicity we shall also assume that both $D$ and $\mathbb{R}^{3} \backslash \bar{D}$ are connected and simply connected. We wish to approximate the total electric field $\mathbf{E}=\mathbf{E}(\mathbf{x})$,

\footnotetext{
*Department of Mathematical Sciences, U. of Delaware, Newark DE 19716-2553 (hsiao@math.udel.edu).

${ }^{\dagger}$ Department of Mathematical Sciences, U. of Delaware, Newark DE 19716-2553 (monk@math.udel.edu).

${ }^{\ddagger}$ IMA, U. of Minnesota, Minneapolis MN 55455 (nigam@ima.umn.edu).
} 


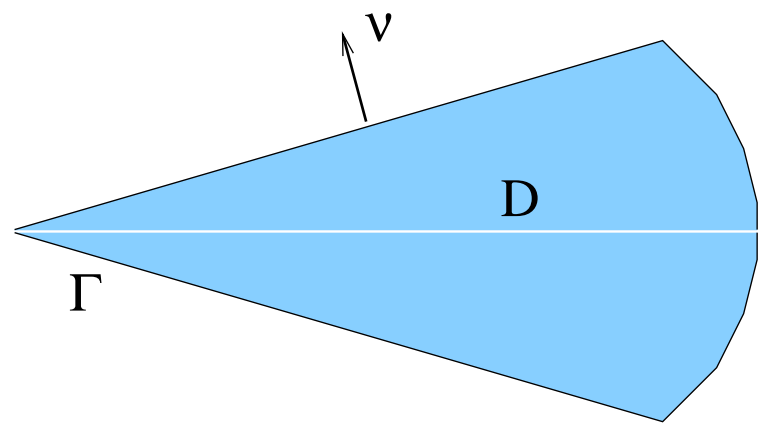

FIG. 1.1. The perfectly conducting scatterer, D.

$\mathbf{x} \in \mathbb{R}^{3} \backslash D$, where this field satisfies the time-harmonic Maxwell's equation:

$$
\nabla \times \nabla \times \mathbf{E}-k^{2} \mathbf{E}=0, \quad \text { in } \mathbb{R}^{3} \backslash \bar{D},
$$

and the boundary condition appropriate for a perfect conductor:

$$
\boldsymbol{\nu} \times \mathbf{E}=0, \quad \text { on } \Gamma .
$$

The real parameter $k>0$ is called the wave number of the time-harmonic field. The total field $\mathbf{E}$ is given by

$$
\mathbf{E}=\mathbf{E}^{i}+\mathbf{E}^{s} \quad \text { in } \mathbb{R}^{3} \backslash D
$$

where the given incident field $\mathbf{E}^{i}$ satisfies Maxwell's equation (1.1) in all of $\mathbb{R}^{3}$, and $\mathbf{E}^{s}$ is the unknown scattered field. A typical choice for the incident field is a plane wave in which case

$$
\mathbf{E}^{i}=\mathbf{p} \exp (i k \mathbf{d} \cdot \mathbf{x})
$$

where the real vectors $\mathbf{p}$ (polarization) and $\boldsymbol{d}$ satisfy $|\mathbf{p}|=|\mathbf{d}|=1$ and $\mathbf{p} \cdot \mathbf{d}=0$.

In order to uniquely determine the scattered field, we need to impose the SilverMüller radiation condition

$$
\lim _{|x| \rightarrow \infty}\left(\nabla \times \mathbf{E}^{s}\right) \times \mathbf{x}-i k|\mathbf{x}| \mathbf{E}_{T}^{s}=0,
$$

uniformly in $\hat{\mathbf{x}}=\mathbf{x} /|\mathbf{x}|$ where

$$
\mathbf{E}_{T}^{s}=\left(\hat{\mathbf{x}} \times \mathbf{E}^{s}\right) \times \hat{\mathbf{x}}
$$

More generally, for a sufficiently smooth vector function $\mathbf{u}$, we use the notation

$$
\mathbf{u}_{T}:=\left(\boldsymbol{\nu} \times\left.\mathbf{u}\right|_{S}\right) \times \boldsymbol{\nu}
$$

to denote the tangential component of $\mathbf{u}$ on a given surface $S$ with normal $\boldsymbol{\nu}$. Equations (1.1)-(1.4) are uniquely solvable in appropriate function spaces which will be given shortly. 


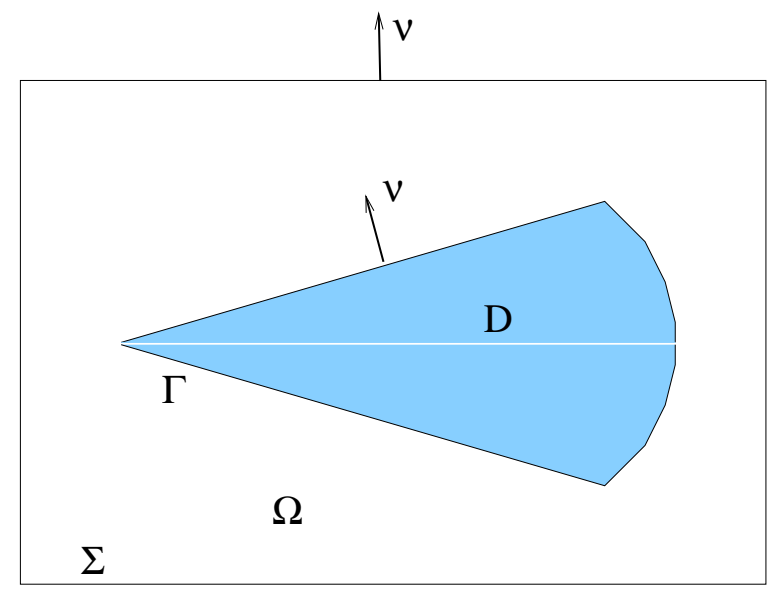

FIG. 1.2. The truncated computational domain, $\Omega:=D_{\Sigma} \backslash D$

1.2. Truncation of problem. As stated in the previous section, the scattering problem is posed on an infinite region. In order to apply a finite element method, we need to truncate the domain. Following Hazard and Lenoir, we introduce a simply connected, Lipschitz, polyhedral surface $\Sigma$, with interior $D_{\Sigma}$, such that $\bar{D} \subset D_{\Sigma}$. The outward unit normal on $\Sigma$ is again denoted $\boldsymbol{\nu}$. For technical reasons associated with the proof of Lemma 3.3, we restrict $\Sigma$ to be a right parallelepiped. We expect that a more general polyhedral surface would also be appropriate. A smooth surface such as a sphere might also be used, but the analysis of such a scheme would involve the use of curvilinear finite elements which is outside the scope of this paper. We define the truncated computational domain

$$
\Omega:=D_{\Sigma} \backslash \bar{D}
$$

Our assumptions imply that the boundary of $\Omega$ consists of two disjoint, connected components $\Sigma$ and $\Gamma$, and the region $\Omega$ is simply connected (see Figure 1.2). The goal is to use finite elements on $\Omega$ to approximate $\mathbf{E}$, but we need a boundary condition on $\Sigma$. This is provided by recalling the Stratton-Chu formula that gives a representation of classical solutions of (1.1)-(1.4) away from $\Gamma$ (see [15]). More precisely, let

$$
\mathbb{G}(\mathbf{x}, \mathbf{y})=\Phi(\mathbf{x}, \mathbf{y}) \mathbb{I}+k^{-2} \operatorname{Hess}(\Phi)(\mathbf{x}, \mathbf{y}),
$$

where $\mathbb{I}$ is the identity matrix, $\Phi(\mathbf{x}, \mathbf{y})$ is the fundamental solution of the Helmholtz equation in $\mathbb{R}^{3}$, given by

$$
\Phi(\mathbf{x}, \mathbf{y}):=\frac{e^{i k|\mathbf{x}-\mathbf{y}|}}{4 \pi|\mathbf{x}-\mathbf{y}|},
$$

and $\operatorname{Hess}(\cdot)$ is the Hessian operator defined by

$$
\operatorname{Hess}(\Phi)_{l, m}=\frac{\partial^{2} \Phi}{\partial y_{l} \partial y_{m}}, \quad 1 \leq l, m \leq 3
$$


For $\mathbf{x} \in \mathbb{R}^{3} \backslash \bar{D}$, we have

$$
\begin{aligned}
\mathbf{E}^{s}(\mathbf{x})= & \int_{\Gamma}\left\{\mathbb{G}(\mathbf{x}, \mathbf{y})^{T}\left(\boldsymbol{\nu}_{y} \times\left(\nabla \times \mathbf{E}^{s}(\mathbf{y})\right)\right)\right. \\
=: & \mathcal{I}\left(\mathbf{E}^{s}\right),
\end{aligned}
$$

where $\nabla_{y} \times \mathbb{G}(\mathbf{x}, \mathbf{y})$ is the column-wise curl with respect to $\mathbf{y}$. Using the fact that $\mathbf{E}^{i}$ is a regular solution of Maxwell's equations inside $D$, we have $\mathcal{I}\left(\mathbf{E}^{i}\right)=0$ in $\Omega$, and thus

$$
\mathbf{E}=\mathbf{E}^{i}+\mathcal{I}(\mathbf{E}) \quad \text { in } \Omega,
$$

provided $\mathbf{E}$ is regular enough for $\mathcal{I}(\mathbf{E})$, defined in (1.5), to hold.

1.3. A modified integral representation. Unfortunately, the regularity requirement implicit in (1.5) is not met by edge finite elements. We therefore need to extend the definition of $\mathcal{I}$ to allow for less regular arguments $\mathbf{E}^{s}$. In order to do this, we first recall that

$$
H(\operatorname{curl} ; \Omega)=\left\{\mathbf{u} \in\left(L^{2}(\Omega)\right)^{3} \mid \nabla \times \mathbf{u} \in\left(L^{2}(\Omega)\right)^{3}\right\},
$$

and define the subspace

$$
X:=\left\{\mathbf{u} \in H(\operatorname{curl} ; \Omega) \mid \boldsymbol{\nu} \times \mathbf{u}=0 \text { on } \Gamma \text {, and } \boldsymbol{\nu} \times\left.\mathbf{u}\right|_{\Sigma} \in\left(L^{2}(\Sigma)\right)^{3}\right\} .
$$

The space $X$ is equipped with the norm

$$
\|\mathbf{u}\|_{X}^{2}=\|\mathbf{u}\|_{H(\operatorname{curl} ; \Omega)}^{2}+\left\|\mathbf{u}_{T}\right\|_{\left(L^{2}(\Sigma)\right)^{3}}^{2},
$$

where $\|\cdot\|_{H(\operatorname{curl} ; \Omega)}$ is the standard norm on $H(\operatorname{curl} ; \Omega)$, and $\|\cdot\|_{\left(L^{2}(\Sigma)\right)^{3}}$ is the $\left(L^{2}(\Sigma)\right)^{3}$ norm.

Let $\chi \in C_{0}^{\infty}\left(D_{\Sigma}\right)$ denote a cutoff function such that $\chi=1$ on $\Gamma$ and define $\widetilde{\mathbb{G}}(\mathbf{x}, \cdot) \in H(\operatorname{curl} ; \Omega)$ by

$$
\widetilde{\mathbb{G}}(\mathbf{x}, \mathbf{y})=\chi(\mathbf{y}) \mathbb{G}(\mathbf{x}, \mathbf{y}) .
$$

We can now define the integral operator

$$
\begin{aligned}
\mathcal{I}^{R}\left(\mathbf{E}^{s}\right):= & \int_{\Omega}\left(\left(\nabla_{y} \times \widetilde{\mathbb{G}}\right)^{T} \nabla \times \mathbf{E}^{s}-k^{2} \widetilde{\mathbb{G}}^{T} \mathbf{E}^{s}\right) d V(\mathbf{y}) \\
& +\int_{\Gamma}\left(\nabla_{y} \times \mathbb{G}\right)^{T} \boldsymbol{\nu}_{y} \times \mathbf{E}^{s} d A(\mathbf{y}),
\end{aligned}
$$

where the curl is again with respect to $\mathbf{y}$ and the integral is evaluated for $\mathbf{x}$ outside the support of the cutoff function $\chi$ (in particular for $\mathbf{x} \in \Sigma$ ). Using integration by parts we can verify that for a smooth solution $\mathbf{E}^{s}$ of (1.1)-(1.4),

$$
\mathcal{I}^{R}\left(\mathbf{E}^{s}\right)=\mathcal{I}\left(\mathbf{E}^{s}\right)
$$

and thus

$$
\mathbf{E}=\mathbf{E}^{i}+\mathcal{I}^{R}(\mathbf{E})
$$


Note also that since $\mathcal{I}^{R}(\mathbf{E})$ is evaluated outside the support of $\chi$ (for example on $\Sigma$ ) a further integration by parts, and the use of the perfect conducting boundary condition on $\Gamma$, shows that

$$
\mathcal{I}^{R}(\mathbf{E})=\int_{\Omega}\left(\nabla \times(\nabla \times \widetilde{\mathbb{G}})-k^{2} \widetilde{\mathbb{G}}\right)^{T} \mathbf{E} d V(\mathbf{y})
$$

This is the form of $\mathcal{I}^{R}$ we shall use for the first part of the analysis.

Before stating the variational problem for Maxwell's equations, we define one further operator. For a sufficiently smooth field $\mathbf{u}$, we can define a tangential boundary operator on $\Sigma$ as follows:

$$
T(\mathbf{u}):=\left.(\nabla \times \mathbf{u})\right|_{\Sigma} \times \boldsymbol{\nu}-i k \mathbf{u}_{T},
$$

where $\mathbf{u}_{T}$ is the tangential component of $\mathbf{u}$ on $\Sigma$. With this notation, the truncated version of problem (1.1)-(1.4) is to find $\mathbf{E} \in X$ such that

$$
\begin{gathered}
\int_{\Omega} \nabla \times \mathbf{E} \cdot \nabla \times \overline{\boldsymbol{\phi}}-k^{2} \mathbf{E} \cdot \overline{\boldsymbol{\phi}} d V \\
-\int_{\Sigma} i k \mathbf{E}_{T} \cdot \overline{\boldsymbol{\phi}}_{T} d A-\int_{\Sigma} T\left(\mathcal{I}^{R}(\mathbf{E})\right) \cdot \overline{\boldsymbol{\phi}}_{T} d A=\int_{\Sigma} T\left(\mathbf{E}^{i}\right) \cdot \overline{\boldsymbol{\phi}}_{T} d A, \quad \forall \boldsymbol{\phi} \in X .
\end{gathered}
$$

Hazard and Lenoir show [25] that problem (1.11) has a unique solution for every $k>0$, and given incident field $\mathbf{E}^{i}$. It is also easy to see that the solution does not depend on the choice of $\chi$. Indeed, equation (1.11) is identical to equation (4.12) of [25], allowing for differences in notation and with $\tau=t=\infty, \xi_{0}=1, \xi=1, \lambda=i k$, and $\zeta^{-1}=1$. The space $X$ is $H_{\tau}^{E}$ from [25] but without the constraint on $\nabla \cdot \mathbf{E}$. As we shall see, this constraint is implied directly by the variational equation (1.11).

We shall use (1.11) as the basis of the finite element method we shall analyze. There are several advantages to this formulation compared to other finite element formulations. One advantage is that unlike methods which use standard absorbing boundary conditions, the convergence of the formulation in this paper can be verified as the mesh size decreases; moreover, the method can easily be applied to a layered medium. Further, compared to a standard coupled finite element - boundary element scheme, the advantage of this method is that no singular integrals must be approximated (since $\mathbf{x} \neq \mathbf{y}$ in (1.5)). The main disadvantages relate to the matrices arising from the discrete problem. We shall discuss these issues more at the end of the paper, but mention here that the matrices have dense blocks.

2. The Finite Element Method. We describe a method based on the tetrahedral edge elements of Nédélec [31] which we summarize next. Results for the hexahedral elements discussed in the same paper follow in the same way, and will not be detailed here. In addition, smooth curved boundaries can be handled using the mapping scheme described in [22], but only for elements of the lowest order.

We suppose that $\Omega$ has been covered by a regular, quasi-uniform mesh $\tau_{h}$, consisting of tetrahedra of maximum diameter $h$. Let $P_{\ell}$ denote the set of all polynomials in $x_{1}, x_{2}$, and $x_{3}$ of maximum degree $\ell$, and $\widetilde{P}_{\ell}$ denote the set of homogeneous polynomials of degree $\ell$ in $x_{1}, x_{2}$ and $x_{3}$. On each tetrahedron $K \in \tau_{h}$, the finite element functions are taken from the set

$$
R_{\ell}=\left(P_{\ell-1}\right)^{3} \oplus S_{\ell}
$$


for some $\ell=1,2, \ldots$, where

$$
S_{\ell}=\left\{\mathbf{p} \in \widetilde{P}_{\ell}^{3} \mid \mathbf{p}(\mathbf{x}) \cdot \mathbf{x}=0, \quad \forall \mathbf{x}\right\} .
$$

Using these basis functions, we can define

$$
V_{h}=\left\{\mathbf{u}_{h} \in H(\operatorname{curl} ; \Omega)\left|\mathbf{u}_{h}\right|_{K} \in R_{\ell}, \quad \forall K \in \tau_{h}\right\},
$$

and

$$
X_{h}=\left\{\mathbf{u}_{h} \in V_{h} \mid \mathbf{u}_{h} \times \boldsymbol{\nu}=0, \text { on } \Gamma\right\} .
$$

Following [2], we can define an interpolation operator. We proceed by recalling the standard degrees of freedom for these tetrahedral elements. Let $K$ be an element and suppose that $\mathbf{u} \in\left(H^{\frac{1}{2}+\epsilon}(K)\right)^{3}, \epsilon>0$ and $\nabla \times \mathbf{u} \in\left(L^{q}(K)\right)^{3}, q>2$. Then the following degrees of freedom on $K$ are well defined. Let $e$ be any edge of $K$ with unit tangent vector $\boldsymbol{\tau}$ and let $\boldsymbol{\nu}$ denote the outward normal to $K$ for any face $f$. Then let

$$
\begin{aligned}
& M_{e}(\mathbf{u})=\left\{\int_{e} \mathbf{u} \cdot \boldsymbol{\tau} q d s, \quad \forall q \in P_{\ell-1}(e), \forall \text { edges } e \text { of } K\right\}, \\
& M_{f}(\mathbf{u})=\left\{\int_{f} \mathbf{u} \times \boldsymbol{\nu} \cdot \mathbf{q} d A, \forall \mathbf{q} \in\left(P_{\ell-2}(f)\right)^{2}, \forall \text { faces } f \text { of } K\right\}, \\
& M_{K}(\mathbf{u})=\left\{\int_{K} \mathbf{u} \cdot \mathbf{q} d V, \forall \mathbf{q} \in\left(P_{\ell-3}(K)\right)^{3}\right\} .
\end{aligned}
$$

The set $M_{\ell}(\mathbf{u}) \cup M_{f}(\mathbf{u}) \cup M_{k}(\mathbf{u})$ is unisolvent for $R_{l}$, and curl-conforming (see [31]). Thus, we can define an interpolant $\pi_{h}$ element-wise, by requiring that $\left.\pi_{h} \mathbf{u}\right|_{K} \in R_{\ell}$ and

$$
M_{e}\left(\mathbf{u}-\pi_{h} \mathbf{u}\right)=M_{f}\left(\mathbf{u}-\pi_{h} \mathbf{u}\right)=M_{K}\left(\mathbf{u}-\pi_{h} \mathbf{u}\right)=\{0\} .
$$

Error estimates can be proved by scaling to a reference element. In [1] it is shown that

$$
\left\|\mathbf{u}-\pi_{h} \mathbf{u}\right\|_{H(\operatorname{curl} ; \Omega)} \leq C h^{h \ell}\left(\|\mathbf{u}\|_{\left(H^{\ell^{\prime}}(\Omega)\right)^{3}}+\|\nabla \times \mathbf{u}\|_{\left(H^{\ell^{\prime}}(\Omega)\right)^{3}}\right)
$$

for any $\ell^{\prime}$ with $\ell \geq \ell^{\prime}>\frac{1}{2}$.

Now suppose that $\mathbf{u}$ is such that $\nabla \times\left.\mathbf{u}\right|_{K} \in\left(P_{\ell}\right)^{3}$ for each element $K \in \tau_{h}$. Then using a standard scaling argument like the one proving the above estimate, and using the equivalence of norms for piece-wise polynomials on the reference element as in the proof of equation (2.4) of [3], we obtain that for $0<\epsilon \leq \frac{1}{2}$,

$$
\left\|\mathbf{u}-\pi_{h} \mathbf{u}\right\| \leq C\left(h^{\frac{1}{2}+\epsilon}\|\mathbf{u}\|_{\left(H^{\frac{1}{2}+\epsilon}(\Omega)\right)^{3}}+h\|\nabla \times \mathbf{u}\|_{\left(L^{2}(\Omega)\right)^{2}}\right) .
$$

For later use we need to discretize the operator $\mathcal{I}^{R}$ defined in (1.8). Let $\widetilde{\mathbb{G}}_{h}(\mathbf{x}, \cdot)$ denote the matrix function such that if $\tilde{\mathbf{g}}_{h, m}(\mathbf{x}, \cdot)$ is the $m$ th column of $\widetilde{\mathbb{G}}_{h}(\mathbf{x}, \cdot)$ and $\tilde{\mathbf{g}}_{m}(\mathbf{x}, \cdot)$ is the $m$ th column of $\widetilde{\mathbb{G}}(\mathbf{x}, \cdot)$, then (recalling that $\mathbf{x} \in \Sigma$ ): 
1. $\tilde{\mathbf{g}}_{h, m}(\mathbf{x}, \cdot) \in V_{h}, \quad 1 \leq m \leq 3$,

2. $\left(\tilde{\mathbf{g}}_{h, m}(\mathbf{x}, \cdot)\right)_{T}$ interpolates $\left(\mathbf{g}_{m}(\mathbf{x}, \cdot)\right)_{T}$ on $\Gamma$ (using edge and face degrees of freedom $(2.2 \mathrm{a})$ and $(2.2 \mathrm{~b}))$,

3. $\left(\tilde{\mathbf{g}}_{h, i}(\mathbf{x}, \cdot)\right)_{T}=0$ on all tetrahedra having a face or edge on $\Sigma$.

Obviously, this discretization of $\widetilde{\mathbb{G}}(\mathbf{x}, \mathbf{y})$ is not uniquely determined. For computational convenience, we use $\left(\tilde{\mathbf{g}}_{h, m}\right), m=1,2,3$, that decay to zero rapidly away from $\Gamma$. This minimizes the support of $\widetilde{\mathbb{G}}_{h}$, and is the reason for discretizing $\widetilde{\mathbb{G}}$.

We can then define the discretized version of the integral operator defined in (1.8), $\mathcal{I}_{h}(\mathbf{u})$, for $\mathbf{u} \in H(\operatorname{curl} \Omega)$ and $\mathbf{x} \in \mathbb{R}^{3} \backslash \bar{D}$ by

$$
\mathcal{I}_{h}(\mathbf{u})(\mathbf{x})=\int_{\Omega}\left(\left(\nabla \times \widetilde{\mathbb{G}}_{h}(\mathbf{x}, \mathbf{y})\right)^{T} \nabla \times \mathbf{u}(\mathbf{y})-k^{2}\left(\widetilde{\mathbb{G}}_{h}(\mathbf{x}, \mathbf{y})\right)^{T} \mathbf{u}(\mathbf{y})\right) d V(\mathbf{y}) .
$$

As long as $\mathbf{x}$ is on $\Sigma, \mathcal{I}_{h}(\mathbf{u})$ is a smooth function of $\mathbf{x}$. Hence $T\left(\mathcal{I}_{h}(\mathbf{u})\right)$ is a well defined and smooth (tangential) vector field on each face on $\Sigma$.

The finite element analogue of (1.11) is to find $\mathbf{E}_{h} \in X_{h}$ such that

$$
\begin{aligned}
& \int_{\Omega} \nabla \times \mathbf{E}_{h} \cdot \nabla \times \overline{\boldsymbol{\phi}}_{h}-k^{2} \mathbf{E}_{h} \cdot \overline{\boldsymbol{\phi}}_{h} d V-\int_{\Sigma}\left(i k \mathbf{E}_{h, T}+T\left(\mathcal{I}_{h}\left(\mathbf{E}_{h}\right)\right)\right) \cdot \overline{\boldsymbol{\phi}}_{h, T} d A \\
= & \int_{\Sigma} T\left(\mathbf{E}^{i}\right) \cdot \overline{\boldsymbol{\phi}}_{h} d A, \quad \forall \phi_{h} \in X_{h} .
\end{aligned}
$$

Unfortunately we have been unable to prove directly that $\mathbf{E}_{h}$ converges to $\mathbf{E}$. Instead we first analyze the convergence of the solution of the following intermediate problem of finding $\tilde{\mathbf{E}}_{h} \in X_{h}$ such that

$$
\begin{aligned}
& \int_{\Omega} \nabla \times \tilde{\mathbf{E}}_{h} \cdot \nabla \times \bar{\phi}_{h}-k^{2} \tilde{\mathbf{E}}_{h} \cdot \overline{\boldsymbol{\phi}}_{h} d V-\int_{\Sigma}\left(i k \tilde{\mathbf{E}}_{h, T}+T\left(\mathcal{I}^{R}\left(\tilde{\mathbf{E}}_{h}\right)\right)\right) \cdot \bar{\phi}_{h, T} d A \\
= & \int_{\Sigma} T\left(\mathbf{E}^{i}\right) \cdot \bar{\phi}_{h} d A, \quad \forall \phi_{h} \in X_{h} .
\end{aligned}
$$

Here the operator $\mathcal{I}^{R}$ is not discretized.

In the next section we shall show that $\tilde{\mathbf{E}}_{h}$ is well defined and converges to the true solution $\mathbf{E}$. In principle we could implement (2.7) but the integral operator $\mathcal{I}^{R}$ would become increasingly more expensive to evaluate as the mesh size decreases since a volume integral over a fixed volume must be evaluated. Hence we prefer to compute with (2.6) since $\mathcal{I}_{h}$ can be constructed to only involve a skin of tetrahedra that share an edge with $\Gamma$.

Another justification of the use of (2.6) is that the solution $\mathbf{E}_{h}$ is independent of the choice of $\widetilde{\mathbb{G}}_{h}$ (provided the conditions mentioned earlier in this section are satisfied). In order to show this, we state the following lemma, which also partially justifies our choice of $\widetilde{\mathbb{G}}_{h}$. The proof of this lemma is straightforward, and is postponed until the Appendix.

LEMMA 2.1. Suppose (2.6) has a unique solution for each $\widetilde{\mathbb{G}}_{h}$ satisfying the requirements (1)-(3) discussed earlier in this section. Then the solution is independent of the choice of $\widetilde{\mathbb{G}}_{h}$.

From this Lemma it suffices to prove existence and uniqueness for a particular choice of $\widetilde{\mathbb{G}}_{h}$, to then conclude the result for any $\widetilde{\mathbb{G}}_{h}$. 
3. Analysis of the scheme. We will prove that as the mesh size $h$ decreases, the solutions of the discrete problem (2.7) approach the exact solution of (1.11). In order to prove this, we first need to carefully describe the function spaces we will be working with. This is done in Subsection 3.1. In Subsection 3.2, we rewrite both the continuous and the discrete problem in a convenient operator form. In Subsection 3.3 , we show that the operator equations are of Fredholm-type. We also demonstrate the convergence (in some suitable norm) of the discrete operators to their continuous analogues as the mesh size decreases.

Subsection 3.5 concerns a collective compactness result. We follow the general approach of [34], in that we verify convergence of some operation using the theory of collectively compact operators. The main property of edge elements relevant to this approach is the discrete compactness property (see [27, 28, 29, 34]). Subsection 3.6 combines all these results into our first theorem about the finite element scheme.

An alternative approach to proving the operator convergence via the theory of mixed methods has been employed by Boffi $[11,10,9]$. So far this approach has been aimed at proving convergence in $\left(L^{2}(\Omega)\right)^{3}$ which is appropriate for eigenvalue problems. In that case Boffi has proved the equivalence of the mixed method and the discrete compactness approaches ([8], see also [14]) so the choice of which method to use is immaterial.

For the remainder of the paper we shall assume that $\Gamma$ and $\Sigma$ are each connected, so $\partial \Omega=\Gamma \cup \Sigma$ and $\Gamma \cap \Sigma=\phi$. We could allow both boundaries to be disconnected if necessary at the cost of more notation and complexity.

3.1. Some function spaces and estimates. In this Subsection, we define useful function spaces, which shall be used in the remainder of the paper. Convenient decompositions and properties of these spaces are also listed. Let

$$
S=\left\{p \in H^{1}(\Omega) \mid p=0 \text { on } \Gamma \text { and } p=\text { constant on } \Sigma\right\} .
$$

Then $\nabla S \subset X$ and is a closed subspace of $X$. Hence we may write, using the $\left(L^{2}(\Omega)\right)^{3}$ inner product,

$$
X=X_{0} \oplus \nabla S
$$

and

$$
X_{0}=\left\{\mathbf{u} \in X \mid \int_{\Omega} \mathbf{u} \cdot \nabla \xi d V=0, \quad \forall \xi \in S\right\}
$$

Using Costabel's regularity result [16] we know that the injection $X_{0} \rightarrow\left(L^{2}(\Omega)\right)^{3}$ is compact. Furthermore suppose $\mathbf{u} \in X_{0}, \nabla \times \mathbf{u}=0$ in $\Omega$ and $\mathbf{u}_{T}=0$ on $\Sigma$. Then since $\nabla \times \mathbf{u}=0$, and $\Omega$ is simply connected, there is a scalar potential $p \in H^{1}(\Omega)$ such that

$$
\mathbf{u}=\nabla p
$$

The tangential components of $\mathbf{u}$ vanish on $\Gamma$ and $\Sigma$, and thus we can take $p \in S$. The fact that $\mathbf{u} \in \nabla S$ and $\mathbf{u} \in X_{0}$ then implies $\mathbf{u}=0$. Thus, using the compactness result above, for $\mathbf{u} \in X_{0}$ there is a constant $C>0$ such that

$$
\|\mathbf{u}\|_{X} \leq C\left(\|\nabla \times \mathbf{u}\|_{\left(L^{2}(\Omega)\right)^{3}}+\left\|\mathbf{u}_{T}\right\|_{\left(L^{2}(\Sigma)^{3}\right)}\right) .
$$


We can also decompose $X_{h}$. It is well known (see [31]) that if

$$
S_{h}=\left\{p_{h} \in S\left|p_{h}\right|_{K} \in P_{\ell}, \forall K \in \tau_{h}\right\}
$$

then

$$
\nabla S_{h} \subset X_{h}
$$

and we may write (again using the $\left(L^{2}(\Omega)\right)^{3}$ inner product)

$$
X_{h}=X_{0, h} \oplus \nabla S_{h}
$$

where

$$
X_{0, h}=\left\{\mathbf{u}_{h} \in X_{h} \mid \int_{\Omega} \mathbf{u}_{h} \cdot \nabla \xi_{h} d V=0, \quad \forall \xi_{h} \in S_{h}\right\}
$$

is the space of discrete divergence free fields. The main difficulty with the analysis of the error is that $X_{0, h} \not \subset X_{0}$.

3.2. An operator equation. In order to use the Fredholm Alternative in the analysis of the finite element formulation, we rewrite the continuous variational problem (1.11) and the discrete finite element problem (2.6) as operator equations. We introduce some convenient notation, to be used in the remainder of this section. For $\mathbf{u}, \mathbf{v} \in X$ we denote

$$
a(\mathbf{u}, \mathbf{v})=\int_{\Omega} \nabla \times \mathbf{u} \cdot \nabla \times \overline{\mathbf{v}}+k^{2} \mathbf{u} \cdot \overline{\mathbf{v}} d V-i k \int_{\Sigma} \mathbf{u}_{T} \cdot \overline{\mathbf{v}}_{T} d A .
$$

Note that $|a(\mathbf{u}, \mathbf{u})|$ is a norm equivalent to $\|\mathbf{u}\|_{X}$. Define the operator $A:\left(L^{2}(\Omega)\right)^{3} \rightarrow$ $\left(L^{2}(\Omega)\right)^{3}$ such that for all $\mathbf{f} \in\left(L^{2}(\Omega)\right)^{3}, A \mathbf{f} \in X_{0} \subset\left(L^{2}(\Omega)\right)^{3}$ satisfies

$$
a(A \mathbf{f}, \boldsymbol{\phi})=-2 k^{2} \int_{\Omega} \mathbf{f} \cdot \bar{\phi} d V-\int_{\Sigma} T\left(\mathcal{I}^{R}(\mathbf{f})\right) \cdot \bar{\phi}_{T} d A, \quad \forall \boldsymbol{\phi} \in X_{0} .
$$

By the Lax-Milgram lemma this problem is well posed. In particular using the expression for $\mathcal{I}^{R}$ in (1.9) shows that

$$
\left\|T\left(\mathcal{I}^{R}(\mathbf{u})\right)\right\|_{L^{2}(\Sigma)} \leq C\|\mathbf{u}\|_{L^{2}(\Omega)}
$$

which allows us to prove the continuity of $a(\cdot, \cdot)$.

Similarly we define $\mathbf{F} \in X_{0}$ by

$$
a(\mathbf{F}, \boldsymbol{\phi})=\int_{\Sigma} T\left(\mathbf{E}^{i}\right) \cdot \bar{\phi} d A, \quad \forall \boldsymbol{\phi} \in X_{0} .
$$

We proceed to show that the operator problem of finding $\mathbf{E} \in\left(L^{2}(\Omega)\right)^{3}$ such that

$$
\mathbf{E}+A \mathbf{E}=\mathbf{F}
$$

is exactly equivalent to solving the Hazard-Lenoir equation (1.11). Any solution of (1.11) is divergence-free, and thus if we pick a test function $\phi \in X_{0}$, then (1.11) can be recast as: find $\mathbf{E} \in X_{0}$ such that

$$
a(\mathbf{E}+A \mathbf{E}-\mathbf{F}, \boldsymbol{\phi})=0, \quad \forall \boldsymbol{\phi} \in X_{0} .
$$


Hence, in $X, \mathbf{E}+A \mathbf{E}-\mathbf{F}=0$ and this certainly implies equality in $\left(L^{2}(\Omega)\right)^{3}$. Conversely, if we have a solution $\mathbf{E} \in\left(L^{2}(\Omega)\right)^{3}$ of

$$
\mathbf{E}+A \mathbf{E}=\mathbf{F}
$$

then since $\mathbf{E}=\mathbf{F}-A \mathbf{E}$, we know that $\mathbf{E} \in X_{0}$. Therefore $\mathbf{E}$ satisfies

$$
a(\mathbf{E}+A \mathbf{E}-\mathbf{F}, \boldsymbol{\xi})=0, \quad \forall \boldsymbol{\xi} \in X,
$$

which is the Hazard-Lenoir equation (1.11). This shows the equivalence of the operator equation (3.12) and the Hazard-Lenoir equation (1.11). The existence and uniqueness of solutions to (3.12) now follow from those of (1.11). (See [25]).

3.3. The Fredholm Alternative. Hazard and Lenoir prove the compactness of $A$ as an operator from $X_{0}$ to $X_{0}$. We need to perform the analysis in $\left(L^{2}(\Omega)\right)^{3}$ since $X_{0, h} \not \subset X_{0}$. In fact, $A$ is compact as a map from $\left(L^{2}(\Omega)\right)^{3}$ to $\left(L^{2}(\Omega)\right)^{3}$ as the next lemma shows.

Lemma 3.1. The map $A:\left(L^{2}(\Omega)\right)^{3} \rightarrow\left(L^{2}(\Omega)\right)^{3}$ is compact.

Proof. By the Lax-Milgram Lemma, $A$ is well defined as a map from $\left(L^{2}(\Omega)\right)^{3}$ into $X_{0}$. The extension of Weber's compactness theorem due to Costabel [16] proves that $X_{0}$ is compactly embedded in $\left(L^{2}(\Omega)\right)^{3}$. This proves the compactness of $A$.

Using this lemma we can see that (3.12) is a Fredholm equation on $\left(L^{2}(\Omega)\right)^{3}$ and hence, via Hazard and Lenoir's uniqueness result, (3.12) has a unique solution $\mathbf{E}$ in $X$.

Now we write the discrete problem (2.7) as an operator equation. We define the operator $\tilde{A}_{h}:\left(L^{2}(\Omega)\right)^{3} \rightarrow\left(L^{2}(\Omega)\right)^{3}$ as the straightforward discrete analogue of $A$. By this we mean that for a given $\mathbf{f} \in\left(L^{2}(\Omega)\right)^{3}, \tilde{A}_{h} \mathbf{f} \in X_{0, h}$ satisfies

$$
a\left(\tilde{A}_{h} \mathbf{f}, \boldsymbol{\xi}_{h}\right)=-2 k^{2} \int_{\Omega} \mathbf{f} \cdot \overline{\boldsymbol{\xi}}_{h} d V-\int_{\Sigma} T\left(\mathcal{I}^{R}(\mathbf{f})\right) \cdot \overline{\boldsymbol{\xi}}_{h, T} d A, \quad \forall \boldsymbol{\xi}_{h} \in X_{0, h}
$$

We can also define $\mathbf{F}_{h} \in X_{0, h}$ by

$$
a\left(\mathbf{F}_{h}, \boldsymbol{\xi}_{h}\right)=\int_{\Sigma} T\left(\mathbf{E}^{i}\right) \cdot \overline{\boldsymbol{\xi}}_{h, T} d A, \quad \forall \boldsymbol{\xi}_{h} \in X_{0, h}
$$

The operator $\tilde{A}_{h}$ and vector $\mathbf{F}_{h}$ are well-defined by the coercivity of $a(\cdot, \cdot)$ and the Lax-Milgram Lemma.

We can then pose the problem of finding $\tilde{\mathbf{E}}_{h} \in\left(L^{2}(\Omega)\right)^{3}$ such that

$$
\tilde{\mathbf{E}}_{h}+\tilde{A}_{h} \tilde{\mathbf{E}}_{h}=\mathbf{F}_{h} .
$$

Assuming such a solution can be found,

$$
\tilde{\mathbf{E}}_{h}=\mathbf{F}_{h}-\tilde{A}_{h} \tilde{\mathbf{E}}_{h} \in X_{0, h} .
$$

As a first step in our analysis of this problem, we now need to demonstrate that as the mesh size $h$ decreases, the discrete operator $\tilde{A}_{h}$ converges to $A$. This is the content of the next lemma, the proof of which is rather classical (see [26]).

Lemma 3.2. For fixed $\mathbf{f} \in\left(L^{2}(\Omega)\right)^{3}, \tilde{A}_{h} \mathbf{f} \rightarrow$ Af in $X$ as $h \rightarrow 0$. 
Proof. We rewrite the problem defining $A,(3.10)$, as the mixed problem of finding $A \mathbf{f} \in X$ and $p \in S$ such that

$$
\begin{aligned}
a(A \mathbf{f}, \boldsymbol{\xi})+ & \int_{\Omega} \boldsymbol{\xi} \cdot \nabla \bar{p} d V=-2 k^{2} \int_{\Omega} \mathbf{f} \cdot \overline{\boldsymbol{\xi}} d V-\int_{\Sigma} T\left(\mathcal{I}_{h}(\mathbf{f})\right) \cdot \overline{\boldsymbol{\xi}}_{T} d A, \quad \forall \boldsymbol{\xi} \in X, \\
& \int_{\Omega} A \mathbf{f} \cdot \nabla \bar{\phi} d V=0, \quad \forall \phi \in S .
\end{aligned}
$$

Since $a(\cdot, \cdot)$ is coercive on $X$ and $\nabla S \subset X$, we can easily verify the Babuška-Brezzi condition and conclude this is a well-posed problem (taking $\boldsymbol{\xi} \in X_{0}$ shows it reduces to (3.10) in this case).

Similarly, the discrete problem (3.13) may be written as

$$
\begin{aligned}
& a\left(\tilde{A}_{h} \mathbf{f}, \boldsymbol{\xi}_{h}\right)+\int_{\Omega} \boldsymbol{\xi}_{h} \cdot \nabla \bar{p}_{h} d V=-2 k^{2} \int_{\Omega} \mathbf{f} \cdot \overline{\boldsymbol{\xi}}_{h} d V-\int_{\Sigma} T\left(\mathcal{I}_{h}(\mathbf{f})\right) \cdot \overline{\boldsymbol{\xi}}_{h, T} d A, \quad \forall \boldsymbol{\xi}_{h} \in X_{h}, \\
& (3.16) \quad \int_{\Omega} \tilde{A}_{h} \mathbf{f} \cdot \nabla \bar{\phi}_{h} d V=0, \quad \forall \phi_{h} \in S_{h} .
\end{aligned}
$$

The coercivity of $a(\cdot, \cdot)$ and the fact that $\nabla S_{h} \subset X_{h}$ allows us to verify the discrete Babuška-Brezzi condition, and conclude that the following estimate holds (see [13]):

$$
\begin{aligned}
& \left\|A \mathbf{f}-\tilde{A}_{h} \mathbf{f}\right\|_{X}+\left\|\nabla\left(p-p_{h}\right)\right\| \\
& \leq c\left\{\inf _{\chi_{h} \in X_{h}}\left\|A \mathbf{f}-\chi_{h}\right\|_{X}+\inf _{\xi_{h} \in S_{h}}\left\|\nabla\left(p-\xi_{h}\right)\right\|\right\} .
\end{aligned}
$$

The theorem follows from standard arguments using the density of $S_{h}$ in $S$ and $X_{h}$ in $X$.

The pointwise convergence of $\tilde{A}_{h}$ to $A$ is not sufficient to conclude that the operator $\left(I+\tilde{A}_{h}\right)$ is invertible. Before proving this invertibility we need to prove a technical regularity result.

3.4. A regularity result. Before stating and proving our main compactness result we need the following regularity result. This result claims that if a vector field $\mathbf{u} \in X$ and a discrete field $\mathbf{u}_{h} \in X_{h}$ have curls which agree in $\Omega$, and if the tangential components of the fields agree on the boundary, then $\mathbf{u}$ possesses some extra regularity. The proof of this lemma proceeds by considering a decomposition of $\mathbf{u}$, and establishing a regularity result for each component.

Lemma 3.3. Let $\mathbf{u}_{h} \in X_{0, h}$ and suppose $\mathbf{u} \in X_{0}$ satisfies

$$
\begin{aligned}
& \nabla \times \mathbf{u}=\nabla \times \mathbf{u}_{h} \text { in } \Omega \\
& \boldsymbol{\nu} \times \mathbf{u}=\boldsymbol{\nu} \times \mathbf{u}_{h} \text { on } \partial \Omega .
\end{aligned}
$$

Then there is an $\epsilon_{\max }>0$ such that $\mathbf{u} \in\left(H^{s}(\Omega)\right)^{3}$, for $1 / 2 \leq s<1 / 2+\epsilon_{\max }$, and

$$
\|\mathbf{u}\|_{\left(H^{s}(\Omega)\right)^{3}} \leq C\left(\|\nabla \times \mathbf{u}\|_{\left(L^{2}(\Omega)\right)^{3}}+\|\boldsymbol{\nu} \times \mathbf{u}\|_{\left(H^{s-1 / 2}(\Sigma)\right)^{3}}\right) .
$$

REMARK 1. (1) In [16] this result is proved for $0 \leq s \leq 1 / 2$, and in [2] the result is proved when $\boldsymbol{\nu} \times \mathbf{u}=0$ on $\partial \Omega$ (including $\Sigma$ ). The result here is possible because 
$\boldsymbol{\nu} \times \mathbf{u}_{h}$ is a piecewise polynomial on $\Gamma$ and $\Sigma$, and hence smoother than just square integrable on $\Gamma$ and $\Sigma$. The proof we shall give combines those in [2] and [16].

(2) It is in the proof of this theorem that we use the fact that $\Sigma$ is a parallelepiped. This is a hypothesis for an extension result given in Lemma 6.2. Note that extension results of this type are valid for arbitrary Lipschitz polygons in $\mathbb{R}^{2}$ (see [24] Theorem 1.5.2.3 for example). Dauge (private communication) suggests that the same is true in $\mathbb{R}^{3}$. Assuming this is so, the proofs in this paper are valid for more general outer boundaries $\Sigma$.

Proof. In this proof we shall use the spaces $H^{l}(\Sigma)$ for $l>0$. We define (here on $\Sigma$, but using obvious notation also on $\Gamma$ )

$$
H^{l}(\Sigma)=\left\{g=\left.\xi\right|_{\Sigma} \mid \xi \in H^{l+1 / 2}(\Omega)\right\}
$$

with the norm

$$
\|g\|_{H^{l}(\Sigma)}=\inf _{\xi \in H^{l+1 / 2}(\Omega),\left.\xi\right|_{\Sigma}=g}\|\xi\|_{H^{l+1 / 2}(\Omega)} .
$$

This definition makes sense for all $l>0$ and agrees with the trace space defined in terms of intrinsic norms for $l \leq 1$.

As in [16], let $\mathcal{O}$ denote a smooth, bounded, connected domain with simply connected boundary $\partial \mathcal{O}$ containing $\bar{\Omega}$ in its interior. First we construct a vector potential $\mathbf{w} \in\left(H^{1}(\mathcal{O})\right)^{3}$ such that

$$
\left.\begin{array}{rl}
\nabla \times \mathbf{w} & =\nabla \times \mathbf{u} \\
\nabla \cdot \mathbf{w} & =0
\end{array}\right\} \text { in } \Omega
$$

Let $\mathbf{z}$ be defined on $\mathcal{O}$ by

$$
\mathbf{z}= \begin{cases}0 & \text { in } D, \\ \nabla \times \mathbf{u} & \text { in } \Omega, \\ \nabla \xi & \text { in } \mathcal{O} \backslash \overline{(\Omega \cup D)}\end{cases}
$$

where $\xi \in H^{1}(\mathcal{O} \backslash \overline{(\Omega \cup D)}) / \mathbb{R}$ solves the boundary value problem

$$
\begin{aligned}
& \Delta \xi=0 \text { in } \mathcal{O} \backslash \overline{(\Omega \cup D)} \\
& \frac{\partial \xi}{\partial \nu}=\boldsymbol{\nu} \cdot \nabla \times \mathbf{u} \text { on } \Sigma \\
& \frac{\partial \xi}{\partial \nu}=0 \text { on } \partial \mathcal{O} .
\end{aligned}
$$

Note that $\boldsymbol{\nu} \cdot \nabla \times \mathbf{u} \in H^{-1 / 2}(\Sigma)$ since $\nabla \cdot(\nabla \times \mathbf{u})=0$, and that this also implies the necessary compatibility condition for solvability.

Of course, $\boldsymbol{\nu} \cdot \nabla \times \mathbf{u}=0$ on $\Gamma$ since the boundary condition is perfectly conducting. Thus $\mathbf{z}$ has a continuous normal component across $\Sigma$ and $\Gamma, \nabla \cdot \mathbf{z}=0$ in $\mathcal{O}$. Hence Lemma 3.5 of [2] ensures that there is a function $\mathbf{w} \in\left(H^{1}(\mathcal{O})\right)^{3}$ such that

$$
\nabla \times \mathbf{w}=\mathbf{z}, \text { and } \nabla \cdot \mathbf{w}=0 \text { in } \mathcal{O} .
$$

Hence $\mathbf{w}$ verifies the desired properties in (3.18).

As $\nabla \times(\mathbf{u}-\mathbf{w})=0$, in $\Omega$ there is a scalar potential $p \in H^{1}(\Omega)$, such that

$$
\mathbf{u}-\mathbf{w}=\nabla p .
$$




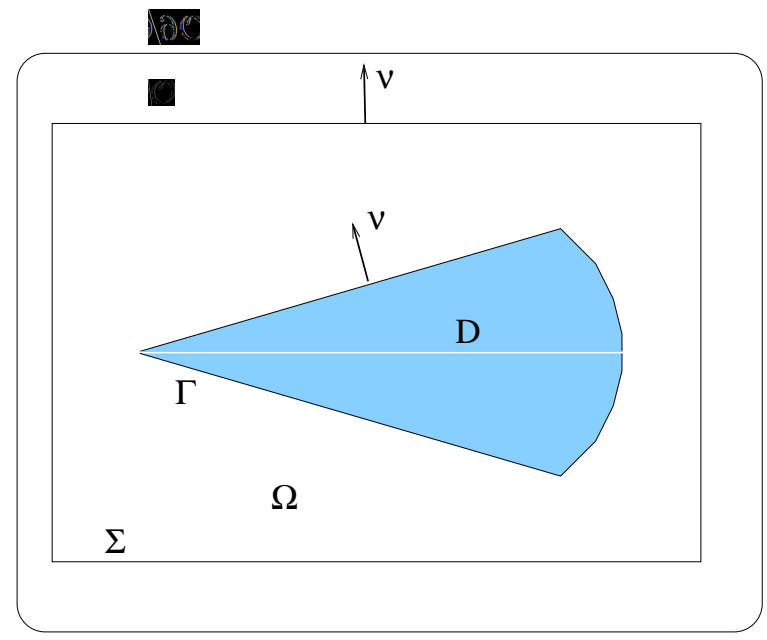

FIG. 3.1. The configuration of the smooth enclosing domain domain $O$.

The fact that $\nabla \cdot(\mathbf{u}-\mathbf{w})=0$ then implies that

$$
\Delta p=0 \text { in } \Omega .
$$

We need to demonstrate that $p$ possesses extra regularity, in order to establish the smoothness of $\mathbf{u}$. This can be done following [2]. Inside $D$ we have $\nabla \times \mathbf{w}=0$, and since $\mathbf{w} \in\left(H^{1}(\mathcal{O})\right)^{3}$, there is a scalar potential $\eta \in H^{2}(D)$, such that $\mathbf{w}=\nabla \eta$ in $D$. On the boundary $\Gamma$, we have

$$
(\boldsymbol{\nu} \times(\mathbf{u}-\mathbf{w})) \times \boldsymbol{\nu}=(\boldsymbol{\nu} \times \nabla p) \times \boldsymbol{\nu} .
$$

We now use the boundary condition on $\Gamma$ and the fact that $\mathbf{w}=\nabla \eta$ in $D$ to obtain

$$
\nabla_{\Gamma} p=-\nabla_{\Gamma} \eta
$$

where we have used the notation $\nabla_{\Gamma} p=(\boldsymbol{\nu} \times \nabla p) \times \boldsymbol{\nu}$ so that $\nabla_{\Gamma}$ is the surface gradient. Since $\eta \in H^{3 / 2}(\Gamma)$, we can conclude $p \in H^{3 / 2}(\Gamma)$.

For the outer boundary $\Sigma$, we follow [16]. Clearly $\left.p\right|_{\Sigma} \in H^{1 / 2}(\Sigma)$, and $\left.\mathbf{w}\right|_{\Sigma} \in$ $\left(H^{1 / 2}(\Sigma)\right)^{3}$. Furthermore on $\Sigma$,

$$
(\boldsymbol{\nu} \times(\mathbf{u}-\mathbf{w})) \times \boldsymbol{\nu}=(\boldsymbol{\nu} \times \nabla p) \times \boldsymbol{\nu} .
$$

Thus, on $\Sigma$,

$$
\mathbf{u}_{T}-\mathbf{w}_{T}=\nabla_{\Sigma} p .
$$

Unlike the boundary condition on $\Gamma$, now $\mathbf{u}_{T}$ does not vanish on $\Sigma$, since

$$
\mathbf{u}_{T}=\mathbf{u}_{h, T}
$$

The polyhedral nature of $\Sigma$ implies that on each face $F$ of the surface, we have that $\boldsymbol{\nu}$ is a constant vector, and $\mathbf{u}_{h}$ is a piecewise polynomial, so

$$
\mathbf{u}_{h, T}=\left(\boldsymbol{\nu} \times \mathbf{u}_{h}\right) \times \boldsymbol{\nu} \in\left(H^{\epsilon}(F)\right)^{3}
$$


for $0 \leq \epsilon<1 / 2$. Thus $\nabla_{\Sigma} p \in\left(H^{\epsilon}(F)\right)^{3}$ and hence $p \in H^{1+\epsilon}(F)$. Moreover since $p \in H^{1 / 2}(\Sigma)$ it cannot have line discontinuities, so in fact $p$ is continuous on $\Sigma$.

We are thus assured that that $p$ is continuous at each edge of $\Sigma$, and $p \in H^{1+\epsilon}(F)$ for each face $F$. Hence via Lemma 6.2 , there is an extension, denoted $\tilde{p} \in H^{3 / 2+\epsilon}(\Omega)$. Then, using this extension, and considering $p-\tilde{p}$ we can conclude, using Corollary 18.15 of Dauge [20], that there is an $\epsilon_{\max }$ with $0<\epsilon_{\max }<1 / 2$ such that $p \in$ $H^{3 / 2+\epsilon}(\Omega)$ for $0 \leq \epsilon<\epsilon_{\max }$. Using the fact that $\mathbf{u}=\mathbf{w}+\nabla p$ completes the proof. $\mathrm{Q}$

3.5. A Collective Compactness result. Let $\Lambda$ be a countable set whose only accumulation point is at zero. We assume that the mesh size $h \in \Lambda$ and hence that there is a sequence of mesh sizes $h_{n} \rightarrow 0$ as $n \rightarrow \infty$.

Lemma 3.4. The set of operators $\left\{\tilde{A}_{h}\right\}_{h \in \Lambda}$ is collectively compact considered as maps from $\left(L^{2}(\Omega)\right)^{3}$ to $\left(L^{2}(\Omega)\right)^{3}$.

REMARK 2. This is essentially the discrete compactness property (for a full discussion of this property see [27, 28, 29, 9]).

Proof. Let $U \subset\left(L^{2}(\Omega)\right)^{3}$ be a bounded set and define

$$
\mathcal{A}(U)=\left\{\mathbf{w} \in X \mid \mathbf{w}=\tilde{A}_{h}(\mathbf{u}) \text { for some } \mathbf{u} \in U \text { and } h \in \Lambda\right\} .
$$

To prove collective compactness, we need to show $\mathcal{A}(U)$ is pre-compact in $\left(L^{2}(\Omega)\right)^{3}$. Let $\left\{\mathbf{w}_{n}\right\} \subset \mathcal{A}(U)$ be a sequence. Then for each $n$ there is an $h_{n}$ and $\mathbf{u}_{n} \in U$ such that

$$
\mathbf{w}_{n}=\tilde{A}_{h_{n}}\left(\mathbf{u}_{n}\right) \in X_{0, h_{n}} .
$$

Without loss of generality we can assume $h_{n} \rightarrow 0$ as $n \rightarrow \infty$ (otherwise we are in a finite dimensional vector space and a convergent subsequence of $\left\{\mathbf{w}_{n}\right\}$ is guaranteed).

Let $p^{n} \in S$ satisfy

$$
\int_{\Omega} \nabla p^{n} \cdot \nabla \bar{\xi} d V=\int_{\Omega} \mathbf{w}_{n} \cdot \nabla \bar{\xi} d V, \quad \forall \xi \in S
$$

We can decompose $\mathbf{w}_{n} \in\left(L^{2}(\Omega)\right)^{3}$ by defining $\mathbf{w}^{n}$ as

$$
\mathbf{w}^{n}=\mathbf{w}_{n}-\nabla p^{n} .
$$

Clearly, $\mathbf{w}^{n} \in X_{0}$. Since $\left\{\mathbf{w}_{n}\right\}$ is a bounded subset of $X$, we can conclude $\left\{\mathbf{w}^{n}\right\}$ is a bounded subset in $X_{0}$. Thus, using the compactness result of [16], there is a subsequence, still denoted $\left\{\mathbf{w}^{n}\right\}$, and an element $\mathbf{w} \in X_{0}$, such that

$$
\mathbf{w}^{n} \longrightarrow \mathbf{w} \text { as } n \rightarrow \infty\left\{\begin{array}{rll}
\text { weakly } & \text { in } X, \\
\text { strongly } & \text { in }\left(L^{2}(\Omega)\right)^{3} .
\end{array}\right.
$$

Moreover, the definition of $\mathbf{w}^{n}$ reveals that since $\mathbf{w}_{n}$ is a finite element function

$$
\nabla \times \mathbf{w}^{n}=\nabla \times \mathbf{w}_{n} \quad \in\left(L^{q}(\Omega)\right)^{3},
$$

and $\boldsymbol{\nu} \times \mathbf{w}^{n}=\boldsymbol{\nu} \times \mathbf{w}_{n}$ on $\Sigma($ and $\Gamma)$.

Hence by Lemma $3.3, \mathbf{w}^{n} \in\left(H^{\frac{1}{2}+\epsilon}(\Omega)\right)^{3}$ for some $\epsilon>0$. Since $\nabla \times \mathbf{w}^{n} \in\left(L^{q}(\Omega)\right)^{3}$ for any $q$, we can conclude that the interpolant $\pi_{h_{n}} \mathbf{w}^{n}$ is well-defined. The interpolant of $\mathbf{w}_{n}$, and hence the interpolant of $\nabla p^{n}$, is also well defined. Thus,

$$
\pi_{h_{n}} \mathbf{w}^{n}=\pi_{h_{n}}\left(\mathbf{w}_{n}-\nabla p^{n}\right)=\mathbf{w}_{n}-\nabla p_{n}
$$


for some $p_{n} \in S_{h_{n}}$. The relation

$$
\pi_{h_{n}} \nabla p=\nabla p_{n} \quad \text { for some } p_{n} \in S_{h_{n}}
$$

follows from properties of edge elements (see [23]). Hence, using the fact that $\mathbf{w} \in X_{0}$ and $\mathbf{w}_{n} \in X_{0, h_{n}}$,

$$
\begin{aligned}
\int_{\Omega}\left(\mathbf{w}-\mathbf{w}_{n}\right) \cdot \overline{\left(\mathbf{w}^{n}-\mathbf{w}_{n}\right)} d V & =\int_{\Omega}\left(\mathbf{w}-\mathbf{w}_{n}\right) \cdot \overline{\left(\mathbf{w}^{n}-\pi_{h_{n}} \mathbf{w}^{n}-\nabla p_{n}\right)} d V \\
& =\int_{\Omega}\left(\mathbf{w}-\mathbf{w}_{n}\right) \cdot \overline{\left(\mathbf{w}^{n}-\pi_{h_{n}} \mathbf{w}^{n}\right)} d V
\end{aligned}
$$

so that

$$
\left\|\mathbf{w}-\mathbf{w}_{n}\right\|_{\left(L^{2}(\Omega)\right)^{3}} \leq\left\|\mathbf{w}-\mathbf{w}^{n}\right\|_{\left(L^{2}(\Omega)\right)^{3}}+\left\|\mathbf{w}^{n}-\pi_{h_{n}} \mathbf{w}^{n}\right\|_{\left(L^{2}(\Omega)\right)^{3}} .
$$

Now we can use the estimate (2.4) to conclude (using the fact that $\nabla \times \mathbf{w}^{n}=\nabla \times \mathbf{w}_{n}$ ) that

$$
\begin{aligned}
\left\|\mathbf{w}-\mathbf{w}_{n}\right\|_{\left(L^{2}(\Omega)\right)^{3}} \leq & \left\|\mathbf{w}-\mathbf{w}^{n}\right\|_{\left(L^{2}(\Omega)\right)^{3}} \\
& +C\left(h^{1 / 2+\epsilon}\left\|\mathbf{w}^{n}\right\|_{\left(H^{1 / 2+\epsilon}(\Omega)\right)^{3}}+h\left\|\nabla \times \mathbf{w}_{n}\right\|_{\left(L^{2}(\Omega)\right)^{3}}\right) .
\end{aligned}
$$

Using this estimate, the a priori estimate in Lemma 3.3, and the following inverse estimate for fractional power Sobolev spaces norms of piecewise polynomial functions on quasi-uniform meshes proved in [12]:

$$
\left\|\boldsymbol{\nu} \times \mathbf{w}_{n}\right\|_{\left(H^{\epsilon}(\Sigma)\right)^{3}} \leq C h^{-\epsilon}\left\|\boldsymbol{\nu} \times \mathbf{w}_{n}\right\|_{\left(L^{2}(\Sigma)\right)^{3}},
$$

we have that

$$
\left\|\mathbf{w}-\mathbf{w}_{n}\right\|_{\left(L^{2}(\Omega)\right)^{3}} \leq\left\|\mathbf{w}-\mathbf{w}^{n}\right\|_{\left(L^{2}(\Omega)\right)^{3}}+C h^{1 / 2}\left\|\mathbf{w}_{n}\right\|_{X} .
$$

The first term on the right hand side tends to zero by construction, and the second term since $h_{n} \rightarrow 0$ as $n \rightarrow \infty$. Hence we have proved the desired result.

3.6. Error estimates. We can now analyze the operator based problems (3.12) and (3.15) which are to find $\mathbf{E} \in\left(L^{2}(\Omega)\right)^{3}$ and $\mathbf{E}_{h} \in\left(L^{2}(\Omega)\right)^{3}$ such that

$$
\begin{aligned}
(I+A) \mathbf{E} & =\mathbf{F}, \\
\left(I+\tilde{A}_{h}\right) \tilde{\mathbf{E}}_{h} & =\mathbf{F}_{h},
\end{aligned}
$$

for $h \in \Lambda$. We have the following theorem:

THEOREM 3.5. For $h \in \Lambda$ sufficiently small, $\left(I+\tilde{A}_{h}\right)^{-1}$ exists and is uniformly bounded as a map from $\left(L^{2}(\Omega)\right)^{3}$ to $\left(L^{2}(\Omega)\right)^{3}$. The following error estimate

$$
\begin{gathered}
\left\|\tilde{\mathbf{E}}_{h}-\mathbf{E}\right\|_{\left(L^{2}(\Omega)\right)^{3}} \leq c\left(\left\|\mathbf{F}-\mathbf{F}_{h}\right\|_{\left(L^{2}(\Omega)\right)^{3}}+\left\|\left(A-\tilde{A}_{h}\right) \mathbf{F}\right\|_{\left(L^{2}(\Omega)\right)^{3}}\right. \\
\left.+\left\|\left(A-\tilde{A}_{h}\right) A \mathbf{E}\right\|_{\left(L^{2}(\Omega)\right)^{3}}\right)
\end{gathered}
$$

holds with $c$ independent of $h, \mathbf{E}$ and $\mathbf{F}$.

Proof. We start with a slight modification of the proof of Theorem 10.9 of [30]. From that theorem we know that if $h \in \Lambda$ is small enough, $\left(I+\tilde{A}_{h}\right)$ is invertible with 
uniformly bounded inverse as a map from $\left(L^{2}(\Omega)\right)^{3}$ to $\left(L^{2}(\Omega)\right)^{3}$ (because $\left\{A_{h}\right\}_{h \in \Lambda}$ is a collectively compact and pointwise convergent sequence of operators). Thus $\tilde{\mathbf{E}}_{h}$ is well defined.

Then

$$
\mathbf{E}-\tilde{\mathbf{E}}_{h}=\left(I+\tilde{A}_{h}\right)^{-1}\left(\mathbf{F}-\mathbf{F}_{h}\right)+\left((I+A)^{-1}-\left(I+\tilde{A}_{h}\right)^{-1}\right) \mathbf{F}
$$

and the following error estimate follows from the bound on $\left((I+A)^{-1}-\left(I+\tilde{A}_{h}\right)^{-1}\right)$ in [30]:

$$
\begin{aligned}
\left\|\tilde{\mathbf{E}}_{h}-\mathbf{E}\right\|_{\left(L^{2}(\Omega)\right)^{3}} \leq & c\left(\left\|\mathbf{F}-\mathbf{F}_{h}\right\|_{\left(L^{2}(\Omega)\right)^{3}}+\left\|\left(A-\tilde{A}_{h}\right) \mathbf{F}\right\|_{\left(L^{2}(\Omega)\right)^{3}}\right. \\
& \left.+\left\|\left(A-\tilde{A}_{h}\right) A \mathbf{E}\right\|_{\left(L^{2}(\Omega)\right)^{3}}\right)
\end{aligned}
$$

TheOrem 3.6. Provided $h \in \Lambda$ is small enough, the discrete variational problem (2.7) has a unique solution $\tilde{\mathbf{E}}_{h} \in X_{h}$. Furthermore

$$
\left\|\tilde{\mathbf{E}}_{h}-\mathbf{E}\right\|_{X} \leq C\left(\inf _{\chi_{h} \in X_{h}}\left\|\mathbf{F}-\chi_{h}\right\|_{X}+\inf _{\phi_{h} \in X_{h}}\left\|A \mathbf{F}-\phi_{h}\right\|_{X}+\inf _{\boldsymbol{\psi}_{h} \in X_{h}}\left\|A \mathbf{E}-\boldsymbol{\psi}_{h}\right\|_{X}\right) .
$$

In general, $\tilde{\mathbf{E}}_{h} \rightarrow \mathbf{E}$ in $X$ as $h \rightarrow 0$.

Proof. From the previous theorem, $\mathbf{E}_{h}$ is proved to exist uniquely, and it remains to estimate the error in $X$. From (3.12) and (3.15),

$$
\begin{aligned}
\left\|\mathbf{E}-\tilde{\mathbf{E}}_{h}\right\|_{X} & =\left\|A \mathbf{E}-\tilde{A}_{h} \tilde{\mathbf{E}}_{h}+\mathbf{F}-\mathbf{F}_{h}\right\|_{X} \\
& \leq\left\|\left(A-\tilde{A}_{h}\right) \mathbf{E}\right\|_{X}+\left\|\tilde{A}_{h}\left(\mathbf{E}-\tilde{\mathbf{E}}_{h}\right)\right\|_{X}+\left\|\mathbf{F}-\mathbf{F}_{h}\right\|_{X}
\end{aligned}
$$

But using the definition of $\tilde{A}_{h}$,

$$
\left\|\tilde{A}_{h}\left(\mathbf{E}-\tilde{\mathbf{E}}_{h}\right)\right\|_{X} \leq C\left\|\mathbf{E}-\tilde{\mathbf{E}}_{h}\right\|_{\left(L^{2}(\Omega)\right)^{3}}
$$

Hence, via the previous theorem (and using the fact that the $\left(L^{2}(\Omega)\right)^{3}$ norm is bounded by the $X$ norm),

$$
\begin{aligned}
\left\|\mathbf{E}-\tilde{\mathbf{E}}_{h}\right\|_{X} \leq C( & \left\|\left(A-\tilde{A}_{h}\right) \mathbf{E}\right\|_{X}+\left\|\mathbf{F}-\mathbf{F}_{h}\right\|_{X} \\
& \left.+\left\|\left(A-\tilde{A}_{h}\right) \mathbf{F}\right\|_{\left(L^{2}(\Omega)\right)^{3}}+\left\|\left(A-\tilde{A}_{h}\right) A \mathbf{E}\right\|_{\left(L^{2}(\Omega)\right)^{3}}\right) .
\end{aligned}
$$

Since $A \mathbf{E}=\mathbf{F}-\mathbf{E}$, this can be rewritten

$$
\left\|\mathbf{E}-\tilde{\mathbf{E}}_{h}\right\|_{X} \leq C\left(\left\|\left(A-\tilde{A}_{h}\right) \mathbf{E}\right\|_{X}+\left\|\mathbf{F}-\mathbf{F}_{h}\right\|_{X}+\left\|\left(A-\tilde{A}_{h}\right) \mathbf{F}\right\|_{\left(L^{2}(\Omega)\right)^{3}}\right) .
$$

Now we can estimate each term. Via the estimates for the mixed method used previously, we get

$$
\left\|\mathbf{F}-\mathbf{F}_{h}\right\|_{X} \leq C \inf _{\chi_{h} \in X_{h}}\left\|\mathbf{F}-\chi_{h}\right\|_{X} .
$$

Since $\mathbf{E} \in X_{0}$, the potential $p_{h}$ in (3.16) vanishes when $\mathbf{F}=\mathbf{E}$. Therefore,

$$
\left\|\left(A-\tilde{A}_{h}\right) \mathbf{E}\right\|_{X} \leq C \inf _{\phi_{h} \in X_{h}}\left\|A \mathbf{E}-\phi_{h}\right\|_{X} .
$$


In the same way, since $\mathbf{F} \in X_{0}$,

$$
\left\|\left(A-\tilde{A}_{h}\right) \mathbf{F}\right\|_{X} \leq C \inf _{\boldsymbol{\xi}_{h} \in X_{h}}\left\|A \mathbf{F}-\boldsymbol{\xi}_{h}\right\|_{X} .
$$

The convergence result now follows from a density argument.

This result can be made more specific provided the solution is regular enough. Let

$$
\begin{gathered}
H^{s}(\operatorname{curl} ; \Omega)=\left\{\mathbf{u} \in\left(H^{s}(\Omega)\right)^{3} \mid \nabla \times \mathbf{u} \in\left(H^{s}(\Omega)\right)^{3}, \boldsymbol{\nu} \times \mathbf{u} \in\left(H^{s}(f)\right)^{3}\right. \\
\text { for each face } f \text { of } \Sigma\}
\end{gathered}
$$

for some $s \geq 0$ with norm

$$
\|\mathbf{u}\|_{H^{s}(\operatorname{curl} ; \Omega)}^{2}:=\|\mathbf{u}\|_{H^{s}(\Omega)}^{2}+\|\nabla \times \mathbf{u}\|_{H^{s}(\Omega)}^{2}+\sum_{f \in \Sigma}\|\boldsymbol{\nu} \times \mathbf{u}\|_{H^{s}(f)}^{2} .
$$

Then, the error estimate of Theorem (3.6) can be written as shown below.

Corollary 3.7. If $\mathbf{F}, A \mathbf{F}, A \mathbf{E} \in H^{s}(\operatorname{curl} ; \Omega)$ for some $s>\frac{1}{2}$, then

$$
\left\|\mathbf{E}-\tilde{\mathbf{E}}_{h}\right\|_{X} \leq c h^{\min (s, \ell)},
$$

where the norm on $X$ is given by (1.6).

REMARK 3. For a Lipschitz polyhedral domain, the best we can generally expect is that the above regularity requirements hold for some $s$ with $\frac{1}{2}<s$ but possibly with $s$ less than 1.

4. The Fully Discrete Problem. The discretization we have considered to this point is not optimal for implementation since $\mathcal{I}^{R}$ is expensive to compute. We prefer to use (2.6) in place of $(2.7)$. Let us define $A_{h}:\left(L^{2}(\Omega)\right)^{3} \rightarrow\left(L^{2}(\Omega)\right)^{3}$ such that if $\mathbf{f} \in\left(L^{2}(\Omega)\right)^{3}$ then $A_{h} \mathbf{f} \in X_{0, h}$ satisfies

$$
a\left(A_{h} \mathbf{f}, \boldsymbol{\xi}_{h}\right)=-2 k^{2} \int_{\Omega} \mathbf{f} \cdot \overline{\boldsymbol{\xi}}_{h} d V-\int_{\Sigma} T\left(\mathcal{I}_{h}(\mathbf{f})\right) \cdot \overline{\boldsymbol{\xi}}_{h, T} d A, \quad \forall \boldsymbol{\xi}_{h} \in X_{0, h} .
$$

Then (2.6) is equivalent to solving

$$
\left(I+A_{h}\right) \mathbf{E}_{h}=\mathbf{F}_{h}
$$

In order to prove convergence we make a specific choice of $\widetilde{\mathbb{G}}_{h}$. We choose $\widetilde{\mathbb{G}}_{h}$ to interpolate $\widetilde{\mathbb{G}}$ on $\Omega$ (as a function of $\mathbf{y}$ ). Using this choice we can prove the following lemma.

Lemma 4.1. There is a constant $C$ such that for any $\mathbf{u} \in X$,

$$
\left\|\left(A_{h}-\tilde{A}_{h}\right) \mathbf{u}\right\|_{X} \leq C h^{l}\|\mathbf{u}\|_{X}
$$

Proof. By the definition of $A_{h}$ and $\tilde{A}_{h}$,

$$
a\left(\left(A_{h}-\tilde{A}_{h}\right) \mathbf{u},\left(A_{h}-\tilde{A}_{h}\right) \mathbf{u}\right)=-\int_{\Sigma} T\left(\mathcal{I}_{h}(\mathbf{u})-\mathcal{I}^{R}(\mathbf{u})\right) \cdot \overline{\left(A_{h}-\tilde{A}_{h}\right) \mathbf{u}} d A .
$$

Thus

$$
\left\|\left(A_{h}-\tilde{A}_{h}\right) \mathbf{u}\right\|_{X} \leq C\left\|T\left(\mathcal{I}_{h}(\mathbf{u})-\mathcal{I}^{R}(\mathbf{u})\right)\right\|_{\left(L^{2}(\Sigma)\right)^{3}} .
$$


However for any derivative $D_{x}$ with respect to $\mathbf{x}$, for any $\mathbf{x} \in \Sigma$,

$$
\begin{aligned}
\left|D_{x}\left(\mathcal{I}_{h}(\mathbf{u})-\mathcal{I}^{R}(\mathbf{u})\right)\right| & =\mid \int_{\Omega}\left(\nabla \times D_{x}\left(\widetilde{\mathbb{G}}_{h}-\widetilde{\mathbb{G}}\right)^{T} \nabla \times \mathbf{u}-k^{2} D_{x}\left(\widetilde{\mathbb{G}}_{h}-\widetilde{\mathbb{G}}\right)^{T} \mathbf{u} d V \mid\right. \\
& \leq\left\|D_{x}\left(\widetilde{\mathbb{G}}_{h}-\widetilde{\mathbb{G}}\right)\right\|_{X}\|\mathbf{u}\|_{X} .
\end{aligned}
$$

But since $D_{x} \mathbb{G}$ is smooth when $\mathbf{x} \neq \mathbf{y}$, and $\widetilde{\mathbb{G}}_{h}$ interpolates $\widetilde{\mathbb{G}}$ we may use the interpolation estimate $(2.3)$ to show that

$$
\left\|D_{x}\left(\widetilde{\mathbb{G}}_{h}-\widetilde{\mathbb{G}}\right)\right\|_{X} \leq C h^{l}
$$

and we are done.

Next we verify that $\left(I+\tilde{A}_{h}\right)$ is invertible as a map from $X$ to $X$.

Lemma 4.2. The operator $\left(I+\tilde{A}_{h}\right)$ is invertible with a uniformly bounded inverse as a map from $X$ to $X$.

Proof. We have already seen that this Lemma holds with $\left(L^{2}(\Omega)\right)^{3}$ in place of $X$. Now let $\mathbf{u} \in\left(L^{2}(\Omega)\right)^{3}$ solve

$$
\mathbf{u}+\tilde{A}_{h} \mathbf{u}=\mathbf{F}
$$

for some $\mathbf{F} \in X$ then since $\mathbf{u}=\mathbf{F}-\tilde{A}_{h} \mathbf{u} \in X$,

$$
\begin{aligned}
\|\mathbf{u}\|_{X} & \leq\|\mathbf{F}\|_{X}+\left\|\tilde{A}_{h} \mathbf{u}\right\|_{X} \\
& \leq\|\mathbf{F}\|_{X}+C\|\mathbf{u}\|_{\left(L^{2}(\Omega)\right)^{3}} \\
& \leq\|\mathbf{F}\|_{X}+C\|\mathbf{F}\|_{\left(L^{2}(\Omega)\right)^{3}} .
\end{aligned}
$$

Thus

$$
\left\|\left(I+\tilde{A}_{h}\right)^{-1} \mathbf{F}\right\|_{X} \leq C\|\mathbf{F}\|_{X}
$$

and we are done.

Now we can prove that (4.2) has a unique solution that is close to the solution $\tilde{\mathbf{E}}_{h}$ of $(2.7)$.

THEOREM 4.3. Equation (4.2) (or equivalently (2.6)) has a unique solution $\mathbf{E}_{h} \in$ $X_{h}$ and furthermore if $\tilde{\mathbf{E}}_{h}$ is the solution of (2.7) then, provided $h$ is sufficiently small,

$$
\left\|\mathbf{E}_{h}-\tilde{\mathbf{E}}_{h}\right\|_{X} \leq C h^{l}\left\|\mathbf{E}_{h}\right\|_{X}
$$

REMARK 4. As a result of this theorem we can conclude that $\mathbf{E}_{h}$ satisfies the error estimates in Theorem 3.6 and Corollary 3.7.

Proof. We have already verified (Lemma 4.2$)$ that $\left(I-\tilde{A}_{h}\right)$ is invertible as a map from $X$ to $X$ and the inverse is uniformly bounded. Since

$$
\mathbf{E}_{h}+\tilde{A}_{h} \mathbf{E}_{h}+\left(A_{h}-\tilde{A}_{h}\right) \mathbf{E}_{h}=\mathbf{F}_{h}
$$

we have

$$
\left(I+C_{h}\right) \mathbf{E}_{h}=\left(I+\tilde{A}_{h}\right)^{-1} \mathbf{F}_{h}
$$

where $C_{h}=\left(I+A_{h}\right)^{-1}\left(A_{h}-\tilde{A}_{h}\right)$ and hence using Lemma $4.1\left\|C_{h}\right\|_{L(X, X)} \leq C h^{l}<1$ for $h$ sufficiently small. This implies that $\left(I+C_{h}\right)$ is invertible with bounded inverse in $X$ and hence $\mathbf{E}_{h}$ exists. 
Furthermore

$$
\left(I+\tilde{A}_{h}\right)\left(\mathbf{E}_{h}-\tilde{\mathbf{E}}_{h}\right)=\left(\tilde{A}_{h}-A_{h}\right) \mathbf{E}_{h}
$$

so that

$$
\left\|\mathbf{E}_{h}-\tilde{\mathbf{E}}_{h}\right\|_{X} \leq C h^{l} .
$$

Thus we can conclude that Theorem 3.6 holds for $\mathbf{E}_{h}$.

Now we shall show why (2.6) helps in the discretization of this problem. Let $\left\{\boldsymbol{\xi}_{i}\right\}_{i=1}^{N_{h}}$ be a basis for $X_{h}$. Usually this basis would be constructed using the degrees of freedom (2.2), but other choices are possible [32]. Then we can express $\mathbf{E}_{h} \in X_{h}$ as

$$
\mathbf{E}_{h}=\sum_{i=1}^{N_{h}} E_{i} \boldsymbol{\xi}_{i}
$$

and we may write the variational equation (2.6) as a matrix equation. Let $\vec{E}=$ $\left(E_{1}, \ldots, E_{N_{h}}\right)^{T}$, and let $S$ and $L$ be $N_{h} \times N_{n}$ matrices with

$$
\begin{aligned}
S_{i, j} & =\int_{\Omega} \nabla \times \boldsymbol{\xi}_{j} \cdot \nabla \times \overline{\boldsymbol{\xi}}_{i}-k^{2} \boldsymbol{\xi}_{j} \cdot \overline{\boldsymbol{\xi}}_{i} d V-i k \int_{\Sigma} \boldsymbol{\xi}_{j, T} \cdot \overline{\boldsymbol{\xi}}_{i, T} d A, \\
L_{i j} & =-\int_{\Sigma} T\left(\mathcal{I}_{h}\left(\boldsymbol{\xi}_{j}\right)\right) \cdot \overline{\boldsymbol{\xi}}_{i} d A .
\end{aligned}
$$

Let $\vec{F}$ be the vector with

$$
F_{j}=\int_{\Sigma} T\left(\mathbf{E}^{i}\right) \cdot \overline{\boldsymbol{\xi}}_{j} d A
$$

Then

$$
(S+L) \vec{E}=\vec{F} .
$$

Our analysis guarantees that $S+L$ is invertible for $h$ sufficiently small, but $S+L$ is not particularly well structured from the point of view of numerical linear algebra. It is non-definite and non-symmetric.

The matrix $S$ is somewhat better behaved than $L$. It is sparse and symmetric (but not Hermitian). It corresponds to the standard discretization of an interior problem, and is also invertible for $h$ sufficiently small. In general, $S$ has $O\left(N_{h}\right)$ non-zero entries.

If we choose $\widetilde{\mathbb{G}}_{h}$ to interpolate zero away from $\Gamma$, then $\mathcal{I}_{h}\left(\boldsymbol{\xi}_{j}\right)$ vanishes when $\boldsymbol{\xi}_{j}$ is zero on all tetrahedra sharing an edge with $\Gamma$. Thus $L_{i j} \neq 0$ only if $\boldsymbol{\xi}_{i}$ is associated with an edge or face on $\Sigma$ and $\boldsymbol{\xi}_{j}$ is associated with a tetrahedron touching $\Gamma$. For a quasi-uniform mesh, we expect $O\left(N_{h}^{\frac{2}{3}}\right)$ edges and faces on $\Sigma$, and $O\left(N_{h}^{\frac{2}{3}}\right)$ tetrahedra to touch $\Gamma$. Hence $L$ has $O\left(N_{h}^{\frac{4}{3}}\right)$ non-zero entries which is far more than $S$ has. Thus $L$ is very expensive to compute and store. This suggests that (4.3) should be solved by an iterative technique (BiCG Stab has worked well for us when applying similar methods to the Helmholtz equation) and then only the action of $L$ needs to be computed. We expect that this can be computed rapidly using the fast multipole method [33] to yield a fast over-all solver. 
5. Conclusion. We have proved convergence of the combined finite element, integral equation technique, under fairly general conditions on the scatterer and the auxiliary boundary. When the domain is well-behaved so that the exact solution is regular, we can even obtain optimal order estimates.

The scheme results in a large dense sub-matrix in the overall matrix for the discrete problem. This suggests the necessity of using the fast multipole scheme to evaluate the integral operator $\mathcal{I}_{h}(\mathbf{f})$. We are now programming the combined scheme and hope to report numerical results and algorithmic details in the near future.

Acknowledgment. The effort of George C. Hsiao and Peter Monk was sponsored by the Air Force Office of Scientific Research, Air Force Materials Command, USAF, under grant number F49620-96-1-0039. The US Government is authorized to reproduce and distribute reprints for governmental purposes notwithstanding any copyright notation thereon. The views and conclusions contained herein are those of the authors and should not be interpreted as necessarily representing the official policies or endorsements, either expressed or implied, of the Air Force Office of Scientific Research or the US Government.

6. Appendix. In this appendix, we provide a proof of Lemma (2.1) and an extension theorem for functions defined on a parallelepiped (Lemma 6.2). Let $\widetilde{\mathbb{G}}_{h}(\mathbf{x}, \cdot)$ denote the matrix function such that if $\tilde{\mathbf{g}}_{h, l}(\mathbf{x}, \cdot)$ is the $l$ th column of $\widetilde{\mathbb{G}}_{h}(\mathbf{x}, \cdot)$ and $\mathbf{g}_{l}(\mathbf{x}, \cdot)$ is the $l$ th column of $\mathbb{G}(\mathbf{x}, \cdot)$, then

a) $\tilde{\mathbf{g}}_{h, l}(\mathbf{x}, \cdot) \in V_{h}, \quad 1 \leq l \leq 3$,

b) $\left(\tilde{\mathbf{g}}_{h, l}(\mathbf{x}, \cdot)\right)_{T}$ interpolates $\left(\mathbf{g}_{l}(x, \cdot)\right)_{T}$ on $\Gamma$ (using edge and face freedoms $(2.2 \mathrm{a})$ and $(2.2 \mathrm{~b}))$,

c) $\left(\tilde{\mathbf{g}}_{h, l}(\mathbf{x}, \cdot)\right)_{T}=0$ on all tetrahedra sharing an edge or face with $\Sigma$.

LEMMA 6.1. Suppose (2.6) has a unique solution for each given $\widetilde{\mathbb{G}}_{h}$ satisfying the properties assumed in Section 2. Then the solution is independent of the choice of $\widetilde{\mathbb{G}}_{h}$.

Proof. Suppose $\mathbf{E}_{h}^{(i)}$ is the solution of (2.6) corresponding to $\widetilde{\mathbb{G}}_{h}^{(i)}, i=1,2$. Let $\mathcal{I}_{h}^{(i)}, i=1,2$ denote the operator in (2.5) using $\widetilde{\mathbb{G}}_{h}^{(i)}$. Since solutions of the finite element formulation (2.6) are unique, It suffices to show that

$$
\mathcal{I}_{h}^{(2)}\left(\mathbf{E}_{h}^{(1)}\right)=\mathcal{I}_{h}^{(1)}\left(\mathbf{E}_{h}^{(1)}\right)
$$

By definition

$$
\begin{aligned}
& \left(\mathcal{I}_{h}^{(2)}\left(\mathbf{E}_{h}^{(1)}\right)-\mathcal{I}_{h}^{(1)}\left(\mathbf{E}_{h}^{(1)}\right)\right)^{T} \\
= & \int_{\Omega}\left\{\left(\nabla \times \mathbf{E}_{h}^{(1)}\right)^{T} \nabla \times\left(\widetilde{\mathbb{G}}_{h}^{(2)}-\widetilde{\mathbb{G}}_{h}^{(1)}\right)-k^{2}\left(\mathbf{E}_{h}^{(1)}\right)^{T}\left(\widetilde{\mathbb{G}}_{h}^{(2)}-\widetilde{\mathbb{G}}_{h}^{(1)}\right)\right\} d V .
\end{aligned}
$$

Now the $l$ th column of $\widetilde{\mathbb{G}}_{h}^{(2)}-\widetilde{\mathbb{G}}_{h}^{(1)}$ is

$$
\left(\widetilde{\mathbb{G}}_{h}^{(2)}-\widetilde{\mathbb{G}}_{h}^{(1)}\right)_{l}=\tilde{\mathbf{g}}_{h, l}^{(2)}-\tilde{\mathbf{g}}_{h, l}^{(1)}
$$

and since $\mathbf{g}_{h, l}^{(j)}, j=1,2$ interpolates $\mathbf{g}_{l}$ on $\Gamma$, the tangential component of the difference vanishes there. Hence $\tilde{\mathbf{g}}_{h, l}^{(2)}-\tilde{\mathbf{g}}_{h, l}^{(1)} \in X_{h}$, and so since $\left(\mathbf{g}_{h, i}^{(2)}-\mathbf{g}_{h, i}^{(1)}\right)_{T}=0$ on $\Sigma$ we have, 
from the definition of $\mathbf{E}_{h}^{(1)}$ in (2.6) and using the test function $\phi_{h}=\overline{\left(\tilde{\mathbf{g}}_{h, l}^{(2)}-\tilde{\mathbf{g}}_{h, l}^{(1)}\right)}$,

$$
\int_{\Omega}\left\{\left(\nabla \times \mathbf{E}_{h}^{(1)}\right) \cdot \nabla \times\left(\tilde{\mathbf{g}}_{h, l}^{(2)}-\tilde{\mathbf{g}}_{h, l}^{(1)}\right)-k^{2} \mathbf{E}_{h}^{(1)} \cdot\left(\tilde{\mathbf{g}}_{h, l}^{(2)}-\tilde{\mathbf{g}}_{h, l}^{(1)}\right)\right\} d V=0,
$$

and so $\mathcal{I}_{h}^{(2)}\left(\mathbf{E}_{h}^{(1)}\right)=\mathcal{I}_{h}^{(1)}\left(\mathbf{E}_{h}^{(1)}\right)$. Thus $\mathbf{E}_{h}^{(1)}$ satisfies (2.6) with $\mathcal{I}_{h}=\mathcal{I}_{h}^{(2)}$. The assumed uniqueness of $\mathbf{E}_{h}^{(2)}$ then implies $\mathbf{E}_{h}^{(2)}=\mathbf{E}_{h}^{(1)}$.

Lemma 6.2. Suppose $\Sigma$ is the surface of a right parallelepiped and that there is a function $u \in H^{1}(\Sigma)$ such that on each face $f$ of $\Sigma, u \in H^{1+\epsilon}(\Sigma), 0 \leq \epsilon<1 / 2$ and that $u$ is continuous at the edges of the face of $\Sigma$. Then there is an extension $u \in H^{3 / 2+\epsilon}(P)$ where $P$ denotes the parallelepiped.

REMARK 5. The proof is similar to that of Theorem 1.5.2.4 of [24].

Proof. By a partition of unity we need only consider the problem in the neighborhood of a corner. Suppose the corner is at $\mathrm{b}(0,0,0)$ and that the planes $x=0$, $y=0$ and $z=0$ meet there. Furthermore suppose we wish to extend $u$ to the octant $x>0, y>0$ and $z>0$.

Let $u=g_{1}$ on the quarter plane $\{0\} \times \mathbb{R}_{+} \times \mathbb{R}_{+}, u=g_{2}$ on the quarter plane $\mathbb{R}_{+} \times\{0\} \times \mathbb{R}_{+}$, and $u=g_{3}$ on the quarter plane $\mathbb{R}_{+} \times \mathbb{R}_{+} \times\{0\}$. We can assume $g_{1}$, $g_{2}$ and $g_{3}$ vanish outside a sphere of radius $R$ around $(0,0,0)$ (because of the partition of unity).

Using the standard extension theorem we can extend $g_{1}$ to a function $\tilde{g}_{1} \in$ $H^{1+\epsilon}(\{0\} \times \mathbb{R} \times \mathbb{R})$. This can then be extended to a function $G_{1} \in H^{3 / 2+\epsilon}\left(\mathbb{R}^{3}\right)$ such that $\left.G_{1}\right|_{x=0}=\tilde{g}_{1}$.

Now consider $v=u-G_{1}$. Clearly $v=0$ on $\{0\} \times \mathbb{R}_{+} \times \mathbb{R}_{+}, v=g_{2}-G_{1}$ on $\mathbb{R}_{+} \times\{0\} \times \mathbb{R}_{+}$and $v=g_{3}-G_{1}$ on $\mathbb{R}_{+} \times \mathbb{R}_{+} \times\{0\}$. In particular $v=0$ on the line $x=0, z=0, y>0$. Define a function $w$ by

$$
w(x, y)= \begin{cases}v(x, y, 0) & \text { if } x>0, y>0 \\ -v(-x, y, 0) & \text { if } x<0, y>0 \\ v(x,-y, 0) & \text { if } x>0, y<0 \\ -v(-x,-y, 0) & \text { if } x<0, y<0\end{cases}
$$

Clearly $w$ is continuous on the whole plane $z=0$ and is antisymmetric in $x$. Since it is continuous we can conclude $w \in H^{1+\epsilon}(\mathbb{R} \times \mathbb{R} \times\{0\})$. Using this as Dirichlet data for solving Laplace's equation (with zero data on the hemisphere of radius $R$ in the upper half space) shows that there is a function $G_{2} \in H^{3 / 2+\epsilon}\left(\mathbb{R} \times \mathbb{R} \times \mathbb{R}_{+}\right)$such that $v=G_{2}$ on the plane $z=0$. The odd symmetry of the data is maintained by the solution and so $G_{2}$ vanishes on the plane $x=0$. Hence if $p=v-G_{2}$ we can conclude $p=0$ on $\{0\} \times \mathbb{R}_{+} \times \mathbb{R}_{+}, p=g_{2}-G_{1}-G_{2}$ on $\mathbb{R}_{+} \times\{0\} \times \mathbb{R}_{+}$and $p=0$ on $\mathbb{R}_{+} \times \mathbb{R}_{+} \times\{0\}$.

Now we again extend $p$ to the plane $y=0$ again by reflection. Let $q$ be defined for $y=0$ by

$$
q(x, z)= \begin{cases}p(x, 0, z) & \text { if } x>0, z>0 \\ -p(-x, 0, z) & \text { if } x<0, z>0 \\ -p(x, 0,-z) & \text { if } x>0, z<0 \\ p(-x, 0,-z) & \text { if } x<0, z<0\end{cases}
$$

Now $q \in H^{1+\epsilon}(\mathbb{R} \times\{0\} \times \mathbb{R})$ and is antisymmetric about the lines $y=z=0$ and $y=$ $x=0$. Thus again using this as boundary data for the Dirichlet problem for Laplace's 
equation (again with zero Dirichlet data on the hemisphere in the appropriate half space), we can conclude that there is a function $G_{3}$ such that $p=\left.G_{3}\right|_{y=0}$ and that $G_{3}$ vanishes on $x=0$ and $z=0$. Thus the function

$$
u=G_{1}+G_{2}+G_{3}
$$

is the required extension with the required smoothness.

\section{REFERENCES}

[1] A. Alonso And A. VAlLi, An optimal domain decomposition preconditioner for low-frequency time-harmonic Maxwell equations, Mathematics of Computations, 68 (1999), pp. 607-631.

[2] C. Amrouche, C. Bernardi, M. Dauge, and V. Girault, Vector potentials in threedimensional nonsmooth domains, Math. Meth. Appl. Sci., 21 (1998), pp. 823-864.

[3] D. Arnold, R. FAlK, And R. Winthur, Multigrid in $h($ div $)$ and $h($ curl $)$, Numerische Mathematik, 85 (2000), pp. 197-217.

[4] F. Assous, P. Ciarlet JR., And E.SonnendrüCker, Resolution of the Maxwell equations in a domain with reentrant corners, RAIRO-Math. Model. Num., 32 (1998), pp. 359-389.

[5] I. BABUS̆KA AND A. Miller, The post-processing approach in the finite element method - Part 1: Calculation of displacements, stresses and other higher derivatives of the displacements, International Journal for numerical methods in engineering, 20 (1984), pp. 1085-1109.

[6] - The post-processing approach in the finite element method - Part 2: The calculation of stress intensity factors, International Journal for Numerical Methods in Engineering., 20 (1984), pp. 1110-1129.

[7] - The post-processing approach in the finite element method - Part 3: A posteriori error estimates and adaptive mesh selection, International Journal for Numerical Methods in Engineering, 20 (1984), pp. 2311-2324.

[8] D. BofFI, Fortin operators and discrete compactness for edge elements, Numer. Math., 87 (2000), pp. 229-246.

[9] - A note on the de Rham complex and a discrete compactness property, Applied Mathematics Letters, 14 (2001), pp. 33-38.

[10] D. Boffi, F. Brezzi, and L. Gastaldi, On the convergence of eigenvalues for mixed formulations. Preprint.

[11] - On the problem of spurious eigenvalues in the approximation of linear elliptic problems in mixed form, Math. Comp., 69 (2000), pp. 121-140.

[12] J. Bramble, J. Pasciak, AND J. XU, The analysis of multigrid algorithms with non-nested spaces or non-inherited quadratic forms, Math. Comp., 56 (1991), pp. 1-34.

[13] S. Brenner And L. Scott, The Mathematical Theorey of Finite Element Methods, SpringerVerlag, 1994.

[14] S. Caorsi, P. Fernandes, and M. Raffetto, On the convergence of Galerkin finite element approximations of electromagnetic eigenproblems, SIAM. J. Numer. Anal., 38 (2000), pp. 580-607.

[15] D. Colton and R. Kress, Inverse Acoustic and Electromagnetic Scattering Theory, no. 93 in Applied Mathematical Sciences, Springer-Verlag, New York, second ed., 1998.

[16] M. Costabel, A remark on the regularity of solutions of Maxwell's equations on Lipschitz domains, Math. Meth. Appl. Sci., 12 (1990), pp. 365-368.

[17] M. Costabel And M. Dauge, Singularities of electromagnetic fields in polyhedral domains, Archive for Rational Mechanics and Analysis, 151 (2000), pp. 221-276. Report available at http://www.maths.univ-rennes1.fr/ costabel/.

[18] J. Coyle AND P. Monk, The finite element approximation of scattering in a layered medium, in Analytical and Computational Methods in Scattering and Applied Mathematic, F. Santosa and I. Stakgold, eds., no. 417 in Research Notes in Mathematics, London, 2000, Chapman \& Hall/CRC, pp. 67-84.

[19] P.-M. CutZach AND C. Hazard, Existence, uniqueness and analyticity properties for electromagnetic scattering in a two-layered medium, Mathematical Methods in the Applied Sciences, 21 (1998), pp. 433-461.

[20] M. Dauge, Elliptic Boundary Value Problems on Corner Domains, vol. 1341 of Lecture Notes in Mathematics, Springer Verlag, Berlin, 1988.

[21] M. Dauge, M. Costabel, And D. Martin, Numerical investigation of a boundary penalization method for Maxwell equations. Report available at http://www . maths . univ-rennes1.fr/ dauge/, 1999. 
[22] F. Dubois, Discrete vector potential representation of a divergence free vector field in three dimensional domains: Numerical analysis of a model problem, SIAM J. Numer. Anal., 27 (1990), pp. 1103-1142.

[23] V. Girault and P.-A. Raviart, Finite Element Approximation of the Navier-Stokes Equations, vol. 749 of Lecture Notes in Mathematics, Springer-Verlag, Berlin, 1979.

[24] P. Grisvard, Elliptic problems in nonsmooth domains, Pitman,London, 1985.

[25] C. Hazard AND M. Lenolr, On the solution of time-harmonic scattering problems for Maxwell's equations, SIAM J. Math. Anal., 27 (1996), pp. 1597-1630.

[26] P. Joly, C. Poirier, J.-E. Roberts, And P. Trouve, A new non-conforming finite element method for computation of electromagnetic guided waves, in Computing methods in applied science and engineering, R. Glowinski, ed., Nova Science Publishers, 1991, pp. 433-444.

[27] F. KIKUCHI, An isomorphic property of two Hilbert spaces appearing in electromagnetism: Analysis by the mixed formulation, Japan J. Appl. Math., 3 (1986), pp. 53-58.

[28] - Mixed and penalty formulations for finite element analysis of an eigenvalue problem in electromagnetism, Comp. Meth. Appl. Mech. Eng., 64 (1987), pp. 509-521.

[29] - On a discrete compactness property for the Nedelec finite elements, J. Fac. Sci. Univ. Tokyo, Sect. 1A, Math, 36 (1989), pp. 479-490.

[30] R. KREss, Linear Integral Equations, Springer-Verlag, Berlin, second ed., 1999.

[31] J. NÉDÉLEC, Mixed finite elements in $\mathbb{R}^{3}$, Numer. Math., 35 (1980), pp. 315-341.

[32] _ Eléments finis mixtes incompressibles pour l'équation de Stokes dans $\mathbb{R}^{3}$, Numer. Math., 39 (1982), pp. 97-112.

[33] V. RokHLin, Rapid solution of integral equations of scattering theory in two dimensions, J. Comput. Phys., 86 (1990), pp. 414-439.

[34] L. Vardapetyan and L. Demkowicz, hp-Adaptive finite elements in electromagnetics, Computers Methods in Applied Mechanics and Engineering, 169 (1999), pp. 331-344.

[35] J. Wheeler, Permafrost thermal design for the trans-Alaska pipeline, in Moving Boundary Problems, D. Wilson, A. Solomon, and P. Boggs, eds., Academic Press, New York, 1978, pp. 267-284. 